\title{
New semi-implicit midpoint rule for zero of monotone mappings in Banach spaces
}

\author{
Yan Tang $^{1}$ (D) . Zhiqing Bao ${ }^{1}$
}

Received: 16 January 2018 / Accepted: 10 July 2018 / Published online: 26 July 2018 (C) The Author(s) 2018

\begin{abstract}
Let $E$ be a nonempty closed uniformly convex and 2-uniformly smooth Banach space with dual $E^{*}$ and $A: E^{*} \rightarrow E$ be Lipschitz continuous monotone mapping with $A^{-1}(0) \neq \emptyset$. A new semi-implicit midpoint rule (SIMR) with the general contraction for monotone mappings in Banach spaces is established and proved to converge strongly to $x^{*} \in E$, where $J x^{*} \in A^{-1}(0)$. Moreover, applications to convex minimization problems, solution of Hammerstein integral equations, and semi-fixed point of a cluster of semi-pseudo mappings are included.
\end{abstract}

Keywords Semi-implicit · Monotone mappings · Zero point ·

Viscosity approximation

Mathematics Subject Classification (2010) 47H09 • 47H10 • 47L25

\section{Introduction}

Let $E$ be a real Banach space with dual $E^{*}$. A normalized duality mapping $J: E \rightarrow$ $2^{E^{*}}$ is defined by

$$
J x=\left\{f^{*} \in E^{*}:\left\langle x, f^{*}\right\rangle=\|x\|^{2}=\left\|f^{*}\right\|^{2}\right\} .
$$

Supported by the National Natural Science Foundation of China (11471059) and Science and Technology Research Project of Chongqing Municipal Education Commission (KJ 1706154)and the Research Project of Chongqing Technology and Business University (KFJJ2017069)

Yan Tang

ttyy7999@163.com

1 College of Mathematics and Statistics, Chongqing Technology and Business University, Chongqing 400067, China 
where $\langle\cdot, \cdot\rangle$ denotes the generalized duality pairing between $E$ and $E^{*}$. It is well known that $E$ is smooth if and only if $J$ is single-valued and if $E$ is uniformly smooth then $J$ is uniformly continuous on bounded subsets of $E$. We shall denote the single-value duality mapping by $j$. The norm on $E$ is said to be uniformly Gâteaux differentiable if for each $y \in S_{1}(0):=\{x \in E:\|x\|=1\}$ the $\lim _{t \rightarrow 0} \frac{\|x+t y\|-\|x\|}{t}$ exists uniformly for $x \in S_{1}(0)$. If the norm of $E$ is uniformly Gâteaux differentiable, then $J$ is uniformly norm to weak* continuous on each bounded subset of $E$.

A significant result of Kato [1], a mapping $A$ with domain $D(A)$ and range $R(A)$ in $E$ is accretive if for each $x, y$ in $D(A)$, there exists $j(x-y) \in J(x-y)$ such that

$$
\langle A x-A y, j(x-y)\rangle \geq 0 .
$$

A mapping $A: D(A) \subset E \rightarrow E^{*}$ is said to be monotone if for each $x, y \in D(A)$, the following inequality holds:

$$
\langle x-y, A x-A y\rangle \geq 0 .
$$

If $E=H$, a Hilbert space, the accretive property is equivalent to the monotonicity of $A$ in the sense of Browder [2] and Minty [3]. Interest in such mappings originate mainly in their firm connection with the existence theory for nonlinear equation of evolution (see, e.g., Browder [2]). An early fundamental result in the theory of accretive operators, due to Browder [2], state the initial value problem of ordinary differential equation

$$
\frac{d u}{d t}=A u ; u(0)=u_{0},
$$

is solvable when $A$ is locally Lipshitzian and accretive on $E$. For obtaining the numerical solution of Eq. 1.1, numerous authors devoted themselves to probing methods of approximating and harvested fruitful results (see, e.g., Mustafa [4], Stephen [5], Sina [6], Chidume [7]). One of the powerful numerical methods for the numerical solution of Eq. 1.1 is implicit midpoint rule(IMR) which generates a iterative sequence $\left\{x_{n}\right\}$ via the relation

$$
\frac{1}{h}\left(x_{n+1}-x_{n}\right)=A\left(\frac{x_{n+1}+x_{n}}{2}\right) .
$$

The sequence $\left\{x_{n}\right\}$ generated by Eq. 1.2 converges to the exact solution of Eq. 1.1 (see e.g., Auzinger [8], Bader [9], Bayreuth [10], Xu[11, 30], Song [31], Cai [32]).

In recent decades, a host of mathematicians concentrated on approximating the solution of $0 \in A u$ (when it exists) which is coincided with the equilibrium state: $\frac{d u}{d t}=0, A u=0$ (see, e.g., Berinde [12], Browder [13], Chidume [14] and the references therein) because a variety of problems, for example, convex optimization, linear programming, monotone inclusions and elliptic differential equations can be formulated as the equilibrium state. Therefore, finding a zero of nonlinear operator $A$ is of important task in approximation theory. In studying the equation $0 \in A u$, where $A$ is a accretive operator on a Hilbert space $H$, Browder [13] introduced an operator $T: H \rightarrow H$ by $T=I-A$ where $I$ is the identity mapping on $H$. The operator $T$ is called pseudo-contractive and the zeros of $A$, if they exist, correspond to the fixed points of $T$. Thus, approximating the solution of $0 \in A x$ is transferred to approximating the fixed points of pseudo-contractive mappings which has been extended to 
real Banach spaces by numerous authors. For strongly pseudo-contractive mappings, Chidume [15] proved the following scheme:

$$
x_{n+1}=\left(1-\alpha_{n}\right) x_{n}+\alpha_{n} T x_{n}, n \geq 0,
$$

converges strongly to the unique fixed point of $T$. By replacing $T$ by $I-A$ in this algorithm, the unique solution of $A u=0$ when $A: E \rightarrow E$ is a strongly accretive and Lipschitz map is easily obtained (see Chidume [16]). Later, the sequence has been generalized and extended in various directions, resulting in flouring areas of research, for numerous authors (see, e.g., Agarwal et al. [17], Berdine [12], Chidume [18], Reich [19]).

However, we have to acknowledge that the technique of converting the inclusion $0 \in A u$ into a fixed point problem for $(I-A): E \rightarrow E$ is not applicable in Banach spaces since, in this case when $A$ is monotone, $A$ maps $E$ into $E^{*}$ and the identity map does not make sense (see, e.g., Chidume [20], Xu [21], Sina [6] and other authors). Consequently, algorithms for approximating solutions of equations $0 \in A u$ when $A: E \rightarrow E^{*}$ is of monotone type in Banach spaces has been focused and explored by increasing authors (see, e.g., Zegeye [22], Ibaraki [23]). Recently, for obtaining the solution of $0 \in A u$ in Banach spaces, Chidume [24] introduced the following scheme to approximate the zero of the maximal monotone mapping:

$$
x_{n+1}=J^{-1}\left(J x_{n}-\alpha_{n} A x_{n}-\alpha_{n} \theta_{n}\left(J x_{n}-J u\right), n \geq 0,\right.
$$

where $J$ is the duality mapping.

Inspired and motivated by the above results, we continue the study of methods of approximating the solution of $0 \in A u$ in Banach spaces much more general than those considered in Chidume [24] and Zegeye [22] and other authors. The idea is to use contractions to regularize the implicit midpoint rule for monotone mappings in Banach spaces which are much more general than Hilbert space and accretive mapping in Chidume [15] and $\mathrm{Xu}$ [21]. Besides the strong convergence theorems for the zero point of monotone mapping, applications to convex minimization problems, solution of Hammerstein integral equations and semi-fixed point of a cluster of semipseudo mappings are included.

\section{Preliminaries}

In the sequel, we shall need the following definitions and results. The space $E$ is said to be smooth if $\rho_{E}(\tau)>0, \forall \tau>0$, and the space $E$ is said to be uniformly smooth if $\lim _{t \rightarrow 0^{+}} \rho_{E}(t)=0$, where $\rho_{E}(\tau)$ is defined by

$$
\rho_{E}(\tau)=\sup \left\{\frac{\|x+y\|-\|x-y\|}{2}-1 ;\|x\|=1,\|y\|=\tau\right\} .
$$

Let $p>1$, the space $E$ is said to be $p$-uniformly smooth if there exists a constant $c>0$ such that $\rho_{E}(t) \leq c t^{p}, t>0$. It is well-known that every $p$-uniformly smooth Banach space is uniformly smooth and $L_{p}$ or $W_{m}^{p}$ is 2 - uniformly smooth if $p \geq 2$. 
Furthermore, from Alber [25], we can get that if $E$ is 2-uniformly smooth, then there exists constant $L_{*}>0$ such that

$$
\|J x-J y\| \leq L_{*}\|x-y\|, \forall x, y \in E .
$$

A mapping $A: D(A) \subset E \rightarrow E^{*}$ is said to be Lipschitz continuous if there exists $L>0$ such that for each $x, y \in D(A)$, the following inequality holds:

$$
\|A x-A y\|_{E^{*}} \leq L\|x-y\|_{E} .
$$

Denote the zero set of $A$ by

$$
A^{-1}(0)=\{z \in D(A): 0 \in A z\} .
$$

A mapping $f: C \rightarrow C$ is called contractive with a coefficient if there exists a constant $\rho \in(0,1)$ such that

$$
\|f(x)-f(y)\| \leq \rho\|x-y\|, \quad \forall x, y \in C .
$$

A Banach limit $\mu$ is a bounded linear functional on $l^{\infty}$ such that

$$
\inf \left\{x_{n} ; n \in \mathbb{N}\right\} \leq \mu(x) \leq \sup \left\{x_{n} ; n \in \mathbb{N}\right\}, \quad \forall x=\left\{x_{n}\right\} \in l^{\infty} .
$$

and $\mu\left(x_{n}\right)=\mu\left(x_{n+1}\right), \quad \forall\left\{x_{n}\right\} \in l^{\infty}$. Let $\left\{x_{n}\right\}$ be a bounded sequence in $E$. Then we can define the real valued continuous convex function $\varphi$ on $E$ by

$$
\varphi(y)=\mu\left\|x_{n}-y\right\|^{2}, \forall y \in E,
$$

then $\varphi(y)$ is convex and continuous, and $\varphi(y) \rightarrow \infty$ as $\|y\| \rightarrow \infty$. If $E$ is reflexive, there exists $z \in C$ such that $\varphi(z)=\min _{y \in C} \varphi(y)$, so we can let the set $C_{\text {min }}$ be

$$
C_{\min }=\left\{z \in C ; \varphi(z)=\min _{y \in C} \varphi(y)\right\} .
$$

It is easy to verify that $C_{\min }$ is nonempty, bounded, closed, and convex subset of $E$.

Lemma 2.1 [26] Let $\alpha$ be a real number, and $\left(x_{0}, x_{1}, \ldots\right) \in l^{\infty}$ such that $\mu_{n}\left(x_{n}\right) \leq \alpha$ for all Banach limits. If $\lim \sup _{n \rightarrow \infty}\left(x_{n+1}-x_{n}\right) \leq 0$, then $\lim \sup _{n \rightarrow \infty} x_{n} \leq \alpha$.

Lemma 2.2 (Tan and $X u[27]$ ) Let $\left\{a_{n}\right\}$ be a sequence of nonnegative real numbers satisfying the following relation:

$$
a_{n+1} \leq\left(1-\theta_{n}\right) a_{n}+\sigma_{n}, \quad n \geq 0,
$$

where $\theta_{n}$ and $\left\{\sigma_{n}\right\}$ are real sequences such that

(i). $\lim _{n \rightarrow \infty} \theta_{n}=0, \Sigma_{n=1}^{\infty} \theta_{n}=\infty$;

(ii). $\quad \lim _{n \rightarrow \infty} \frac{\sigma_{n}}{\theta_{n}} \leq 0$ or $\sum_{n=0}^{\infty} \sigma_{n}<\infty$.

Then the sequence $\left\{a_{n}\right\}$ converges to 0 . 
Lemma 2.3 (e.g., Xu [21]) Let $E$ be a real Banach space with dual $E^{*} . J: E \rightarrow 2^{E^{*}}$ be the generalized duality pairing, then for $\forall x, y \in E$,

$$
\|x+y\|^{2} \leq\|x\|^{2}+2\langle y, j(x+y)\rangle, \forall j(x+y) \in J(x+y) .
$$

\section{Main results}

Let $E$ be a nonempty closed uniformly convex and 2-uniformly smooth Banach space with dual $E^{*}$. Let $A: E^{*} \rightarrow E$ be a Lipschitz continuous monotone mapping. For the rest of paper, $\left\{\omega_{n}\right\},\left\{\alpha_{n}\right\},\left\{\beta_{n}\right\},\left\{\gamma_{n}\right\}$ are real sequences in $[0,1]$ satisfying the following conditions:

(i) : $\alpha_{n}+\beta_{n}+\gamma_{n}=1 ; \lim _{n \rightarrow \infty} \alpha_{n}=0 ; \Sigma_{n=1}^{\infty} \alpha_{n}=\infty$;

(ii): $\lim _{n \rightarrow \infty} \frac{\omega_{n}}{\alpha_{n}}=0$ and $\Sigma_{n=0}^{\infty} \omega_{n}<\infty$;

(iii): $f$ is a piecewise function: $f\left(x^{*}\right)=x^{*}$, if $x^{*} \in(A J)^{-1}(0)$; otherwise $f\left(x^{*}\right)$ is a contractive function with coefficient $\rho$.

Theorem 3.1 Let E be a nonempty closed uniformly convex and 2-uniformly smooth Banach space with dual $E^{*}$. Assume that $A: E^{*} \rightarrow E$ is a L-Lipshitz continuous monotone mapping such that $A^{-1}(0) \neq \varnothing$. Let $\left\{x_{n}\right\}$ be a sequence generated by $x_{0} \in E$,

$$
x_{n+1}=\alpha_{n} f\left(x_{n}\right)+\beta_{n} x_{n}+\gamma_{n}\left(I-\omega_{n} A J\right)\left(\frac{x_{n}+x_{n+1}}{2}\right),
$$

where $J$ is the normalized duality mapping. Suppose that $C_{\min } \cap(A J)^{-1}(0) \neq \emptyset$, then the sequence $\left\{x_{n}\right\}$ converges strongly to an element $x^{*} \in(A J)^{-1}(0)$.

Proof First we prove that $\left\{x_{n}\right\}$ is bounded. Since $\alpha_{n} \rightarrow 0$ and $\lim _{n \rightarrow \infty} \frac{\omega_{n}}{\alpha_{n}}=0$ as $n \rightarrow \infty$, there exists $N_{0}>0$ such that $\alpha_{n} \leq \frac{1}{6}, \frac{\omega_{n}}{\alpha_{n}} \leq \frac{1}{9 L L_{*}}, \forall n>N_{0}$. We take $x^{*} \in(A J)^{-1}(0)$ or $J x^{*} \in A^{-1}(0)$. Let $r>0$ be sufficiently large that $x_{N_{0}} \in B_{r}\left(x^{*}\right)$ and $f\left(x_{N_{0}}\right) \in B_{\frac{r}{4}}\left(x^{*}\right)$.

We show that $\left\{x_{n}\right\}$ belongs to $B:=\overline{B_{r}\left(x^{*}\right)}$ for all integers $n \geq N_{0}$. First, it is clearly by construction that $x_{N_{0}} \in B$. We assume that $x_{n} \in B, f\left(x_{n}\right) \in \overline{B_{\frac{r}{4}}\left(x^{*}\right)}, n>$ $N_{0}$, next we prove that $x_{n+1} \in B$. If $x_{n+1}$ does not belong to $B$, then we have that $\left\|x_{n+1}-x^{*}\right\|>r$. From the recursion (3.1) we obtain that

$$
x_{n+1}-x_{n}=\alpha_{n}\left(f\left(x_{n}\right)-x_{n}\right)+\frac{\gamma_{n}}{2}\left(x_{n+1}-x_{n}\right)-\gamma_{n} \omega_{n} A J\left(\frac{x_{n}+x_{n+1}}{2}\right),
$$

by inducing,

$$
\left(1-\frac{\gamma_{n}}{2}\right)\left(x_{n+1}-x_{n}\right)=\alpha_{n}\left(f\left(x_{n}\right)-x_{n}\right)-\gamma_{n} \omega_{n} A J\left(\frac{x_{n}+x_{n+1}}{2}\right),
$$


which means that

$$
x_{n+1}-x_{n}=\frac{2 \alpha_{n}}{2-\gamma_{n}}\left(f\left(x_{n}\right)-x_{n}\right)-\frac{2 \gamma_{n} \omega_{n}}{2-\gamma_{n}} A J\left(\frac{x_{n}+x_{n+1}}{2}\right),
$$

therefore, from Eq. 3.2 and Lemma 2.3 and the fact $x_{n+1}-x^{*}=x_{n+1}-x_{n}+x_{n}-x^{*}$,

$$
\begin{aligned}
\left\|x_{n+1}-x^{*}\right\|^{2}= & \left\|x_{n+1}-x_{n}+x_{n}-x^{*}\right\|^{2} \\
\leq & \left\|x_{n}-x^{*}\right\|^{2}+2\left\langle x_{n+1}-x_{n}, j\left(x_{n+1}-x^{*}\right)\right\rangle \\
= & \left\|x_{n}-x^{*}\right\|^{2}+2\left\langle\frac{2 \alpha_{n}}{2-\gamma_{n}}\left(f\left(x_{n}\right)-x_{n}\right)-\frac{2 \gamma_{n} \omega_{n}}{2-\gamma_{n}} A J\left(\frac{x_{n}+x_{n+1}}{2}\right),\right. \\
& \left.j\left(x_{n+1}-x^{*}\right)\right\rangle \\
= & \left\|x_{n}-x^{*}\right\|^{2}+2\left\langle\frac{2 \alpha_{n}}{2-\gamma_{n}}\left(f\left(x_{n}\right)-x_{n}\right)-\frac{2 \gamma_{n} \omega_{n}}{2-\gamma_{n}} A J\left(\frac{x_{n}+x_{n+1}}{2}\right)\right. \\
& \left.+\frac{2 \alpha_{n}}{2-\gamma_{n}}\left(x_{n+1}-x^{*}\right)-\frac{2 \alpha_{n}}{2-\gamma_{n}}\left(x_{n+1}-x^{*}\right), j\left(x_{n+1}-x^{*}\right)\right\rangle .
\end{aligned}
$$

Furthermore,

$$
\begin{aligned}
\left\|x_{n+1}-x^{*}\right\|^{2}= & \left\|x_{n}-x^{*}\right\|^{2}-2 \frac{2 \alpha_{n}}{2-\gamma_{n}}\left\|x_{n+1}-x^{*}\right\|^{2}+2\left\langle\frac{2 \alpha_{n}}{2-\gamma_{n}}\left(f\left(x_{n}\right)-x^{*}\right)\right. \\
& \left.-\frac{2 \gamma_{n} \omega_{n}}{2-\gamma_{n}} A J\left(\frac{x_{n}+x_{n+1}}{2}\right)+\frac{2 \alpha_{n}}{2-\gamma_{n}}\left(x_{n+1}-x_{n}\right), j\left(x_{n+1}-x^{*}\right)\right\rangle \\
= & \left\|x_{n}-x^{*}\right\|^{2}-2 \frac{2 \alpha_{n}}{2-\gamma_{n}}\left\|x_{n+1}-x^{*}\right\|^{2}+2\left\langle\frac{2 \alpha_{n}}{2-\gamma_{n}}\left(f\left(x_{n}\right)-x^{*}\right)\right. \\
& -\frac{2 \gamma_{n} \omega_{n}}{2-\gamma_{n}} A J\left(\frac{x_{n}+x_{n+1}}{2}\right)+\frac{2 \alpha_{n}}{2-\gamma_{n}}\left(\frac{2 \alpha_{n}}{2-\gamma_{n}}\left(f\left(x_{n}\right)-x_{n}\right)\right. \\
& \left.\left.-\frac{2 \gamma_{n} \omega_{n}}{2-\gamma_{n}} A J\left(\frac{x_{n}+x_{n+1}}{2}\right)\right), j\left(x_{n+1}-x^{*}\right)\right\rangle,
\end{aligned}
$$

that is

$$
\begin{aligned}
\left\|x_{n+1}-x^{*}\right\|^{2} \leq & \left\|x_{n}-x^{*}\right\|^{2}-\frac{4 \alpha_{n}}{2-\gamma_{n}}\left\|x_{n+1}-x^{*}\right\|^{2}+2\left\langle\frac{2 \alpha_{n}}{2-\gamma_{n}}\left(f\left(x_{n}\right)-x^{*}\right)\right. \\
& -\frac{2 \gamma_{n} \omega_{n}}{2-\gamma_{n}} A J\left(\frac{x_{n}+x_{n+1}}{2}\right)+\frac{2 \alpha_{n}}{2-\gamma_{n}}\left(\frac{2 \alpha_{n}}{2-\gamma_{n}}\left(f\left(x_{n}\right)-x^{*}\right)\right. \\
& \left.\left.-\frac{2 \alpha_{n}}{2-\gamma_{n}}\left(x_{n}-x^{*}\right)-\frac{2 \gamma_{n} \omega_{n}}{2-\gamma_{n}} A J\left(\frac{x_{n}+x_{n+1}}{2}\right)\right), j\left(x_{n+1}-x^{*}\right)\right\rangle \\
\leq & \left\|x_{n}-x^{*}\right\|^{2}-\frac{4 \alpha_{n}}{2-\gamma_{n}}\left\|x_{n+1}-x^{*}\right\|^{2}+2\left[\left(\frac{2 \alpha_{n}}{2-\gamma_{n}}+\frac{4 \alpha_{n}^{2}}{\left(2-\gamma_{n}\right)^{2}}\right)\left\|f\left(x_{n}\right)-x^{*}\right\|\right. \\
& +\frac{4 \alpha_{n}^{2}}{\left(2-\gamma_{n}\right)^{2}}\left\|x_{n}-x^{*}\right\|+\left(\frac{4 \alpha_{n} \gamma_{n} \omega_{n}}{\left(2-\gamma_{n}\right)^{2}}+\frac{2 \gamma_{n} \omega_{n}}{2-\gamma_{n}}\right) \| A J\left(\frac{x_{n}+x_{n+1}}{2}\right) \\
& \left.-A J x^{*} \|\right]\left\|x_{n+1}-x^{*}\right\| .
\end{aligned}
$$

Since $\left\|x_{n+1}-x^{*}\right\|>\left\|x_{n}-x^{*}\right\|$, thus we get

$$
\begin{aligned}
\frac{2 \alpha_{n}}{2-\gamma_{n}}\left\|x_{n+1}-x^{*}\right\| \leq & \left(\frac{2 \alpha_{n}}{2-\gamma_{n}}+\frac{4 \alpha_{n}^{2}}{\left(2-\gamma_{n}\right)^{2}}\right)\left\|f\left(x_{n}\right)-x^{*}\right\| \\
& +\frac{4 \alpha_{n}^{2}}{\left(2-\gamma_{n}\right)^{2}}\left\|x_{n}-x^{*}\right\|+\left(\frac{4 \alpha_{n} \gamma_{n} \omega_{n}}{\left(2-\gamma_{n}\right)^{2}}+\frac{2 \gamma_{n} \omega_{n}}{2-\gamma_{n}}\right) \| A J\left(\frac{x_{n}+x_{n+1}}{2}\right) \\
& -A J x^{*} \| .
\end{aligned}
$$


Moreover, since $A$ is $L$-Lipshitz continuous and $J$ is $L_{*}$-Lipschitz continuous,

$$
\begin{aligned}
\left\|x_{n+1}-x^{*}\right\| \leq & \left(1+\frac{2 \alpha_{n}}{2-\gamma_{n}}\right)\left\|f\left(x_{n}\right)-x^{*}\right\| \\
& +\frac{2 \alpha_{n}}{2-\gamma_{n}}\left\|x_{n}-x^{*}\right\|+\left(\frac{2 \gamma_{n} \omega_{n}}{\alpha_{n}}+\frac{\gamma_{n} \omega_{n}}{\alpha_{n}}\right)\left\|A J\left(\frac{x_{n}+x_{n+1}}{2}\right)-A J x^{*}\right\| \\
\leq & \left(1+2 \alpha_{n}\right)\left\|f\left(x_{n}\right)-x^{*}\right\|+2 \alpha_{n}\left\|x_{n}-x^{*}\right\| \\
& +\frac{3 \omega_{n}}{\alpha_{n}} L L_{*}\left(\frac{\left\|x_{n+1}-x^{*}\right\|}{2}+\frac{\left\|x_{n}-x^{*}\right\|}{2}\right) .
\end{aligned}
$$

In view of the assumptions of the parameters $\alpha_{n}$ and $\omega_{n}$, by inducing, we have that

$$
\left\|x_{n+1}-x^{*}\right\| \leq r .
$$

This is contradiction. Therefore, we can get that $\left\{x_{n}\right\}$ belongs to $B$ for all integers $n \geq N_{0}$, which implies the sequence $\left\{x_{n}\right\}$ is bounded, so the sequence $\left\{f\left(x_{n}\right)\right\}$ and $\left\{A J x_{n}\right\}$ are bounded.

Consequently, it is easy to see that $\left\|x_{n+1}-x_{n}\right\| \rightarrow 0$ because $\alpha_{n} \rightarrow 0$ and $\omega_{n}=o\left(\alpha_{n}\right)$

$$
\left\|x_{n+1}-x_{n}\right\| \leq \frac{2 \alpha_{n}}{2-\gamma_{n}}\left\|f\left(x_{n}\right)-x_{n}\right\|+\frac{2 \gamma_{n} \omega_{n}}{2-\gamma_{n}}\left\|A J\left(\frac{x_{n}+x_{n+1}}{2}\right)\right\| \rightarrow 0 .
$$

Next we show that $\lim _{n \rightarrow \infty} \sup \left\langle x^{*}-f\left(x_{n}\right), j\left(x^{*}-x_{n+1}\right)\right\rangle \leq 0$, where $x^{*} \in C_{\min } \cap$ $(A J)^{-1}(0)$.

Since the sequences $\left\{x_{n}\right\}$ and $\left\{f\left(x_{n}\right)\right\}$ are bounded, there exists $R>0$ sufficiently large such that $f\left(x_{n}\right), x_{n} \in B:=\overline{B_{R}\left(x^{*}\right)}, \forall n \in N$. Furthermore, the set $B$ is a bounded closed and convex nonempty subset of $E$. By the convexity of $B$, we have that $(1-t) x^{*}+t f\left(x_{n}\right) \in B$. Then, it follows from the definition of $\varphi$ that $\varphi\left(x^{*}\right) \leq \varphi\left((1-t) x^{*}+t f\left(x_{n}\right)\right)$. Using Lemma 2.3, we have that

$$
\left\|x_{n}-x^{*}-t\left(f\left(x_{n}\right)-x^{*}\right)\right\|^{2} \leq\left\|x_{n}-x^{*}\right\|^{2}-2 t\left\langle f\left(x_{n}\right)-x^{*}, j\left(x_{n}-x^{*}-t\left(f\left(x_{n}\right)-x^{*}\right)\right)\right\rangle,
$$

thus taking Banach limit over $n \geq 1$,

$\mu_{n}\left\|x_{n}-x^{*}-t\left(f\left(x_{n}\right)-x^{*}\right)\right\|^{2} \leq \mu_{n}\left\|x_{n}-x^{*}\right\|^{2}-2 t \mu_{n}\left\langle f\left(x_{n}\right)-x^{*}, j\left(x_{n}-x^{*}-t\left(f\left(x_{n}\right)-x^{*}\right)\right)\right\rangle$, which means that

$$
\begin{aligned}
2 t \mu_{n}\left\langle f\left(x_{n}\right)-x^{*}, j\left(x_{n}-x^{*}-t\left(f\left(x_{n}\right)\right.\right.\right. & \left.\left.\left.-x^{*}\right)\right)\right\rangle \leq \mu_{n}\left\|x_{n}-x^{*}\right\|^{2}-\mu_{n} \| x_{n}-x^{*}-t\left(f\left(x_{n}\right)\right. \\
& \left.-x^{*}\right) \|^{2}=\varphi\left(x^{*}\right)-\varphi\left(x^{*}+t\left(f\left(x_{n}\right)-x^{*}\right)\right) \leq 0,
\end{aligned}
$$

that is

$$
\mu_{n}\left\langle f\left(x_{n}\right)-x^{*}, j\left(x_{n}-x^{*}-t\left(f\left(x_{n}\right)-x^{*}\right)\right)\right\rangle \leq 0 .
$$

By using the weakly lower semi-continuity of the norm on $E$, we get that as $t \rightarrow 0$,

$$
\left\langle f\left(x_{n}\right)-x^{*}, j\left(x_{n}-x^{*}\right)\right\rangle-\left\langle f\left(x_{n}\right)-x^{*}, j\left(x_{n}-x^{*}-t\left(f\left(x_{n}\right)-x^{*}\right)\right)\right\rangle \rightarrow 0 .
$$

Thus, $\forall \varepsilon>0$, there exists $\delta>0$ such that $\forall t \in(0, \delta), n \geq 1$

$$
\left\langle f\left(x_{n}\right)-x^{*}, j\left(x_{n}-x^{*}\right)\right\rangle<\left\langle f\left(x_{n}\right)-x^{*}, j\left(x_{n}-x^{*}-t\left(f\left(x_{n}\right)-x^{*}\right)\right)\right\rangle+\varepsilon,
$$

therefore

$$
\mu_{n}\left\langle f\left(x_{n}\right)-x^{*}, j\left(x_{n}-x^{*}\right)\right\rangle<\mu_{n}\left\langle f\left(x_{n}\right)-x^{*}, j\left(x_{n}-x^{*}-t\left(f\left(x_{n}\right)-x^{*}\right)\right)\right\rangle+\varepsilon .
$$


In view of the arbitrariness of $\varepsilon$, we have that

$$
\mu_{n}\left\langle f\left(x_{n}\right)-x^{*}, j\left(x_{n}-x^{*}\right)\right\rangle \leq 0 .
$$

Since the norm of $E$ is uniformly Gâteaux differentiable, hence $J$ is uniformly norm to weak* continuous on each bounded subset of $E$, then we have that

$$
\lim _{n \rightarrow \infty}\left(\left\langle f\left(x_{n}\right)-x^{*}, j\left(x_{n+1}-x^{*}\right)\right\rangle-\left\langle f\left(x_{n}\right)-x^{*}, j\left(x_{n}-x^{*}\right)\right\rangle\right)=0 .
$$

Thus, the sequence $\left\{\left\langle f\left(x_{n}\right)-x^{*}, j\left(x_{n}-x^{*}\right)\right\rangle\right\}$ satisfies the condition of the Lemma 2.1 , so we have that

$$
\limsup _{n \rightarrow \infty}\left\langle f\left(x_{n}\right)-x^{*}, j\left(x_{n+1}-x^{*}\right)\right\rangle \leq 0 .
$$

Next we show that $\left\|x_{n+1}-x^{*}\right\| \rightarrow 0$.

From Eqs. 3.1, 3.2 and Lemma 2.3, we have that

$$
\begin{aligned}
\left\|x_{n+1}-x^{*}\right\|^{2}= & \left\|x_{n+1}-x_{n}+x_{n}-x^{*}\right\|^{2} \\
= & \left\|x_{n}-x^{*}+\frac{2 \alpha_{n}}{2-\gamma_{n}}\left(f\left(x_{n}\right)-x_{n}\right)-\frac{\gamma_{n} \omega_{n}}{2-\gamma_{n}} A J\left(\frac{x_{n}+x_{n+1}}{2}\right)\right\|^{2} \\
= & \left\|\left(1-\frac{2 \alpha_{n}}{2-\gamma_{n}}\right)\left(x_{n}-x^{*}\right)+\frac{2 \alpha_{n}}{2-\gamma_{n}}\left(f\left(x_{n}\right)-x^{*}\right)-\frac{\gamma_{n} \omega_{n}}{2-\gamma_{n}} A J\left(\frac{x_{n}+x_{n+1}}{2}\right)\right\|^{2} \\
\leq & \left(1-\frac{2 \alpha_{n}}{2-\gamma_{n}}\right)^{2}\left\|x_{n}-x^{*}\right\|^{2}+2\left\langle\frac{2 \alpha_{n}}{2-\gamma_{n}}\left(f\left(x_{n}\right)-x^{*}\right)-\frac{\gamma_{n} \omega_{n}}{2-\gamma_{n}} A J\left(\frac{x_{n}+x_{n+1}}{2}\right),\right. \\
& \left.j\left(x_{n+1}-x^{*}\right)\right\rangle \\
\leq & \left(1-\frac{2 \alpha_{n}}{2-\gamma_{n}}\right)\left\|x_{n}-x^{*}\right\|^{2}+2\left\langle\frac{2 \alpha_{n}}{2-\gamma_{n}}\left(f\left(x_{n}\right)-x^{*}\right)-\frac{\gamma_{n} \omega_{n}}{2-\gamma_{n}} A J\left(\frac{x_{n}+x_{n+1}}{2}\right),\right. \\
& \left.j\left(x_{n+1}-x^{*}\right)\right\rangle .
\end{aligned}
$$

In view of the fact that the sequence $\left\{x_{n}\right\}$ is bounded, without loss of generality, we assume that $M:=\sup \left\{\left\|x_{n}-x^{*}\right\|\right\}$, therefore,

$$
\begin{aligned}
\left\|x_{n+1}-x^{*}\right\|^{2} & \leq\left(1-\frac{2 \alpha_{n}}{2-\gamma_{n}}\right)\left\|x_{n}-x^{*}\right\|^{2}+2 \frac{2 \alpha_{n}}{2-\gamma_{n}}\left\langle\left(f\left(x_{n}\right)-x^{*}\right), j\left(x_{n+1}-x^{*}\right)\right\rangle \\
& +\frac{2 \gamma_{n} \omega_{n}}{2-\gamma_{n}}\left\|A J\left(\frac{x_{n}+x_{n+1}}{2}\right)-A J x^{*}\right\|\left\|x_{n+1}-x^{*}\right\| \\
& \leq\left(1-\frac{2 \alpha_{n}}{2-\gamma_{n}}\right)\left\|x_{n}-x^{*}\right\|^{2}+\sigma_{n},
\end{aligned}
$$

where $\sigma_{n}=\frac{4 \alpha_{n}}{2-\gamma_{n}}\left\langle\left(f\left(x_{n}\right)-x^{*}\right), j\left(x_{n+1}-x^{*}\right)\right\rangle+\frac{2 \gamma_{n} \omega_{n}}{2-\gamma_{n}} L L_{*} M^{2}$.

Putting $\theta_{n}=\frac{2 \alpha_{n}}{2-\gamma_{n}}$, from Lemma 2.2, we shall obtain that

$$
\lim _{n \rightarrow \infty}\left\|x_{n+1}-x^{*}\right\|=0
$$

which means that the consequence $\left\{x_{n}\right\}$ converges strongly to $x^{*}$. The proof is complete.

Theorem 3.2 Let E be a nonempty closed uniformly convex and 2-uniformly smooth Banach space with dual $E^{*}$. Assume that $A: E^{*} \rightarrow E$ is a L-Lipshitz continuous 
monotone mapping such that $A^{-1}(0) \neq \varnothing$. Let $\left\{x_{n}\right\}$ be a sequence generated by $x_{0} \in E$

$$
x_{n+1}=\alpha_{n} f\left(x_{n}\right)+\left(1-\alpha_{n}\right)\left(I-\omega_{n} A J\right)\left(\frac{x_{n}+x_{n+1}}{2}\right),
$$

where $J$ is the normalized duality mapping. Suppose that $C_{\min } \cap(A J)^{-1}(0) \neq \emptyset$, then the sequence $\left\{x_{n}\right\}$ converges strongly to an element $x^{*} \in(A J)^{-1}(0)$..

Proof Putting $\beta_{n}=0$ in Theorem 3.1, the result holds.

In Hilbert spaces, we can see that the duality mapping $J$ is the identity operator. Next, we recall the example of duality mapping $J$ from Alber and Ryazantseva [33].

Example 3.3 (Alber and Ryazantseva [33]) In $\ell^{p}$ spaces, $1<p<\infty$, for arbitrary $x=\left(x_{1}, x_{2}, x_{3}, \ldots\right)$,

$$
J x=\|x\|_{\ell^{p}}^{2-p}\left\{x_{1}\left|x_{1}\right|^{p-2}, x_{2}\left|x_{2}\right|^{p-2}, x_{3}\left|x_{3}\right|^{p-2}, \ldots\right\} .
$$

Theorem 3.4 Let E be a nonempty closed uniformly convex and 2-uniformly smooth Banach space with dual $E^{*}$. Assume that $A: E^{*} \rightarrow E$ is a L-Lipshitz continuous monotone mapping such that $A^{-1}(0) \neq \emptyset$. Let $\left\{x_{n}\right\}$ be a sequence generated by $x_{0} \in E$

$$
x_{n+1}=\alpha_{n} f\left(x_{n}\right)+\beta_{n} x_{n}+\left(1-\alpha_{n}\right)\left(I-\omega_{n} A J\right) x_{n},
$$

where $J$ is the normalized duality mapping. Suppose that $C_{\min } \cap(A J)^{-1}(0) \neq \emptyset$, then the sequence $\left\{x_{n}\right\}$ converges strongly to an element $x^{*} \in(A J)^{-1}(0)$.

If we take $f \equiv u$ a constant, then we have the following corollaries:

Corollary 3.5 Let E be a nonempty closed uniformly convex and 2-uniformly smooth Banach space with dual $E^{*}$. Assume that $A: E^{*} \rightarrow E$ is a L-Lipshitz continuous monotone mapping such that $A^{-1}(0) \neq \emptyset$. Let $u$ be an arbitrary constant, $\left\{x_{n}\right\}$ be a sequence generated by $x_{0} \in E$

$$
x_{n+1}=\alpha_{n} u+\left(1-\alpha_{n}\right)\left(I-\omega_{n} A J\right)\left(\frac{x_{n}+x_{n+1}}{2}\right),
$$

where $J$ is the normalized duality mapping. Suppose that $C_{\min } \cap(A J)^{-1}(0) \neq \emptyset$, then the sequence $\left\{x_{n}\right\}$ converges strongly to an element $x^{*} \in(A J)^{-1}(0)$.

Corollary 3.6 (Zegeye [22]) Let E be a nonempty closed uniformly convex and 2uniformly smooth Banach space with dual $E^{*}$. Assume that $A: E^{*} \rightarrow E$ is a L-Lipshitz continuous monotone mapping such that $A^{-1}(0) \neq \emptyset$. Let $u$ be an arbitrary constant, $\left\{x_{n}\right\}$ be a sequence generated by $x_{0} \in E$

$$
x_{n+1}=\alpha_{n} u+\left(1-\alpha_{n}\right)\left(I-\omega_{n} A J\right) x_{n},
$$

where $J$ is the normalized duality mapping and $\left\{\omega_{n}\right\},\left\{\alpha_{n}\right\}$ are sequences of nonnegative real numbers in $[0,1]$ and

(i). $\lim _{n \rightarrow \infty} \alpha_{n}=0, \Sigma_{n=1}^{\infty} \alpha_{n}=\infty$;

(ii). $\lim _{n \rightarrow \infty} \frac{\omega_{n}}{\alpha_{n}}=0$. 
Suppose that $C_{\min } \cap(A J)^{-1}(0) \neq \emptyset$, then the sequence $\left\{x_{n}\right\}$ converges strongly to an element $x^{*} \in(A J)^{-1}(0)$.

Very recently, Zegeye [22] introduced semi-pseudo mapping $T:=J-A$, where $A: E \rightarrow E^{*}$ is a monotone mapping. The zero points of $A$ (if they exist) are called the semi-fixed points of $T$, that is, the semi-fixed point set of $T$ is denoted by $F_{J}(T):=\{x \in E, T x=J x\}$. If $A: E^{*} \rightarrow E$ is a monotone mapping, then $T=J^{-1}-A$ is semi-pseudo contractive mapping, and the semi-fixed point set of $T$ is denoted by $F_{J}(T):=\{x \in E, T J x=x\}$.

Corollary 3.7 Let E be a nonempty closed uniformly convex and 2-uniformly smooth Banach space with dual $E^{*}$. Assume that $T: E^{*} \rightarrow E$ is a L-Lipschitz continuous semi-pseudo mapping such that $F_{J}(T) \neq \emptyset$ and $A:=J^{-1}-T$ is maximal monotone mapping. Let $\left\{x_{n}\right\}$ be a sequence generated by $x_{0} \in E$

$$
x_{n+1}=\alpha_{n} f\left(x_{n}\right)+\beta_{n} x_{n}+\gamma_{n}\left(\left(1-\omega_{n}\right) I+\omega_{n} T J\right)\left(\frac{x_{n}+x_{n+1}}{2}\right),
$$

where $J$ is the normalized duality mapping. Suppose that $C_{\min } \cap F_{J}(T) \neq \emptyset$, then the sequence $\left\{x_{n}\right\}$ converges strongly to an element $x^{*} \in F_{J}(T)$.

Corollary 3.8 Let E be a nonempty closed uniformly convex and 2-uniformly smooth Banach space with dual $E^{*}$. Assume that $T: E^{*} \rightarrow E$ is a L-Lipshitz continuous semi-pseudo mapping such that $F_{J}(T) \neq \emptyset$ and $A:=J^{-1}-T$ is maximal monotone mapping. Let $\left\{x_{n}\right\}$ be a sequence generated by $x_{0} \in E$

$$
x_{n+1}=\alpha_{n} u+\beta_{n} x_{n}+\gamma_{n}\left(\left(1-\omega_{n}\right) I+\omega_{n} T J\right)\left(\frac{x_{n}+x_{n+1}}{2}\right),
$$

where $J$ is the normalized duality mapping. Suppose that $C_{\min } \cap F_{J}(T) \neq \emptyset$, then the sequence $\left\{x_{n}\right\}$ converges strongly to an element $x^{*} \in F_{J}(T)$.

Example 3.9 (see e.g. Chidume [34]) Let $1<q<p<\infty$ and let $\lambda \in \mathbb{R}$ be arbitrary. Define $T: \ell^{p} \rightarrow \ell^{q}$ by

$$
T x=\left(\lambda, x_{2}, x_{3}, \ldots\right),
$$

then $T$ is semi-pseudo contractive mapping and $x_{\lambda}:=(\lambda, 0,0,0, \ldots)$ is the semifixed points of $T$.

\section{Applications}

\subsection{Application to minimization problems}

In this section, we study the problem of finding a minimizer of a continuously Fréchet differentiable convex functional in Banach spaces. Consider the following minimization problem

$$
\min _{x \in C} g(x)
$$


where $C$ is a nonempty closed convex subset of $E$, and $g: C \rightarrow R$ is a convex and Fréchet-differentiable functional. The sub-differential of the functional $g$ is denote by $\nabla g$ :

$$
\nabla g(x):=\left\{x^{*} \in C: g(y)-g(x) \geq\left\langle y-x, x^{*}\right\rangle, \forall y \in E\right\} .
$$

Clearly, $\nabla g: E \rightarrow 2^{E}$ is a continuous and monotone operator and $0 \in \nabla g\left(x_{0}\right)$ if and only if $x_{0}$ is a minimizer of $g$. Applying Theorem 3.1 and Theorem 3.2, we get the results below.

Theorem 4.1 Let E be a nonempty closed uniformly convex and 2-uniformly smooth Banach space with dual $E^{*}$. Assume that $g: E^{*} \rightarrow(-\infty,+\infty)$ is a L-Lipshitz continuous Fréchet convex differentiable functional such that the gradient of $g, \nabla g$ is Lipschitz continuous monotone mapping with $(\nabla g)^{-1}(0) \neq \emptyset$. Let $\left\{x_{n}\right\}$ be a sequence generated by $x_{0} \in E$

$$
x_{n+1}=\alpha_{n} f\left(x_{n}\right)+\beta_{n} x_{n}+\gamma_{n}\left(I-\omega_{n} \nabla g J\right)\left(\frac{x_{n}+x_{n+1}}{2}\right),
$$

where $J$ is the normalized duality mapping. Suppose that $C_{\min } \cap(\nabla g J)^{-1}(0) \neq \emptyset$, then the sequence $\left\{x_{n}\right\}$ converges strongly to an element $x^{*} \in(\nabla g J)^{-1}(0)$.

Theorem 4.2 Let E be a nonempty closed uniformly convex and 2-uniformly smooth Banach space with dual $E^{*}$. Assume that $g: E^{*} \rightarrow(-\infty,+\infty)$ is a L-Lipshitz continuous Fréchet convex differentiable functional such that the gradient of $g, \nabla g$ is Lipschitz continuous monotone mapping with $(\nabla g)^{-1}(0) \neq \emptyset$. Let $\left\{x_{n}\right\}$ be a sequence generated by $x_{0} \in E$

$$
x_{n+1}=\alpha_{n} f\left(x_{n}\right)+\left(1-\alpha_{n}\right)\left(I-\omega_{n} \nabla g J\right)\left(\frac{x_{n}+x_{n+1}}{2}\right),
$$

where $J$ is the normalized duality mapping. Suppose that $C_{\min } \cap(\nabla g J)^{-1}(0) \neq \emptyset$, then the sequence $\left\{x_{n}\right\}$ converges strongly to an element $x^{*} \in(\nabla g J)^{-1}(0)$.

\subsection{Application to solution of Hammerstein integral equations}

An integral equation (generally nonlinear) of Hammerstein type has the form

$$
u(x)+\int_{\Omega} k(x, y) f(y, u(y))=w(x),
$$

where the unknown function $u$ and inhomogeneous function $w$ lie in a Banach space $E$ of measurable real-valued functions.

By simple transformation, Eq. (4.1) shall be written as

$$
u+K F u=w,
$$

which can be illustrated, without loss of generality, as

$$
u+K F u=0 \text {. }
$$


For the case of real Hilbert space $H$, for $F, K: H \rightarrow H$, Chidume and Zegeye [28] defined an auxiliary map on Cartesian product $E:=H \times H, T: E \rightarrow E$ by

$$
T[u, v]=[F u-v, K v+u] .
$$

It is known that

$T[u, v]=0 \Leftrightarrow u$ is the solution of Eq. 4.2 and $v=F u$.

They obtained strong convergence of an iterative algorithm defined in Cartesian product space $E$ to a solution of Hammerstein equation (4.2). In Banach spaces more general than Hilbert spaces, Chidume and Idu [24] introduced the operator $T: E \times$ $E^{*} \rightarrow E^{*} \times E$

$$
T[u, v]=\left[J u-F u+v, J_{*} v-K v-u\right],
$$

where $F: E \rightarrow E^{*}$ and $K: E^{*} \rightarrow E$ are monotone mappings. They proved that the mapping $A:=J-T$ is monotone and $u^{*}$ is a solution (when is exists) of Hammerstein equation $u+K F u=0$ if and only if $\left(u^{*}, v^{*}\right)$ is zero point of $A$, where $v^{*}=F u^{*}$. Abiding by the construction of the operator $T$, combining with our semi-implicit midpoint rule, the following theorems are obtained.

Theorem 4.3 Assume the Hammerstein equation (4.2) is solvable. Let $E$ be a nonempty closed uniformly convex and 2-uniformly smooth Banach space with dual $E^{*}$. Let $F: E^{*} \rightarrow E, K: E \rightarrow E^{*}$ be Lipshitz continuous monotone mappings. Let $f: C \rightarrow C$ be a contraction with a coefficient $\rho \in(0,1)$. For $\left(x_{0}, y_{0}\right),\left(u_{0}, v_{0}\right) \in E \times E^{*}$, define the sequences $\left\{u_{n}\right\}$ and $\left\{v_{n}\right\}$ respectively by

$$
\begin{aligned}
& u_{n+1}=\alpha_{n} f\left(u_{n}\right)+\beta_{n} u_{n}+\gamma_{n}\left(\frac{u_{n}+u_{n+1}}{2}-\omega_{n}\left(F J\left(\frac{u_{n}+u_{n+1}}{2}\right)-v_{n}\right)\right), \\
& v_{n+1}=\alpha_{n} f\left(v_{n}\right)+\beta_{n} v_{n}+\gamma_{n}\left(\frac{v_{n}+v_{n+1}}{2}-\omega_{n}\left(K J\left(\frac{v_{n}+v_{n+1}}{2}\right)+u_{n}\right)\right),
\end{aligned}
$$

where $\left\{\omega_{n}\right\},\left\{\alpha_{n}\right\},\left\{\beta_{n}\right\},\left\{\gamma_{n}\right\}$ are sequences of nonnegative real numbers in $[0,1]$ and (i): $\quad \alpha_{n}+\beta_{n}+\gamma_{n}=1$;

(ii): $\quad \lim _{n \rightarrow \infty} \alpha_{n}=0, \Sigma_{n=1}^{\infty} \alpha_{n}=\infty$;

(iii): $\lim _{n \rightarrow \infty} \frac{\omega_{n}}{\alpha_{n}}=0$,

then the sequences $\left\{u_{n}\right\}$ and $\left\{v_{n}\right\}$ converge strongly to $u^{*}$ and $v^{*}$ respectively, where $u^{*}$ is a solution of $u+K F u=0$ with $v^{*}=F u^{*}$.

Theorem 4.4 Assume the Hammerstein equation (4.2) is solvable. Let E be a nonempty closed uniformly convex and 2-uniformly smooth Banach space with dual $E^{*}$. Let $F: E^{*} \rightarrow E, K: E \rightarrow E^{*}$ be Lipschitz continuous monotone mappings. Let $u, v \in E$ be arbitrary constant, for $\left(x_{0}, y_{0}\right),\left(u_{0}, v_{0}\right) \in E \times E^{*}$, define the sequences $\left\{u_{n}\right\}$ and $\left\{v_{n}\right\}$ respectively by

$$
u_{n+1}=\alpha_{n} u+\beta_{n} u_{n}+\gamma_{n}\left(\frac{u_{n}+u_{n+1}}{2}-\omega_{n}\left(F J\left(\frac{u_{n}+u_{n+1}}{2}\right)-v_{n}\right)\right) \text {, }
$$




$$
v_{n+1}=\alpha_{n} v+\beta_{n} v_{n}+\gamma_{n}\left(\frac{v_{n}+v_{n+1}}{2}-\omega_{n}\left(K J\left(\frac{v_{n}+v_{n+1}}{2}\right)+u_{n}\right)\right),
$$

where $\left\{\omega_{n}\right\},\left\{\alpha_{n}\right\},\left\{\beta_{n}\right\},\left\{\gamma_{n}\right\}$ are sequences of nonnegative real numbers in $[0,1]$ and (i): $\quad \alpha_{n}+\beta_{n}+\gamma_{n}=1$;

(ii): $\quad \lim _{n \rightarrow \infty} \alpha_{n}=0, \Sigma_{n=1}^{\infty} \alpha_{n}=\infty$;

(iii): $\lim _{n \rightarrow \infty} \frac{\omega_{n}}{\alpha_{n}}=0$,

then the sequences $\left\{u_{n}\right\}$ and $\left\{v_{n}\right\}$ converge strongly to $u^{*}$ and $v^{*}$ respectively, where $u^{*}$ is a solution of $u+K F u=0$ with $v^{*}=F u^{*}$.

\subsection{Application to a cluster of semi-pseudo mappings}

Let $C$ be a nonempty convex subset of a real Banach space. Let $\left\{T_{i}, i=1,2, \cdots, N\right\}$ be a finite of semi-pseudo mappings. Define

$$
T=\Sigma_{i=1}^{N} s_{i} T_{i}
$$

where $s_{i}, i=1,2, \cdots, N$ are sequences in $[0,1]$ and $\Sigma_{i=1}^{N} s_{i}=1$. We can prove that $T$ is semi-pseudo and $J-T$ is monotone mapping.

Lemma 4.5 Let $C$ be a nonempty closed convex and bounded subset of a smooth Banach space $E$ and $\left\{T_{i}: C \rightarrow C, i=1,2, \cdots, N\right\}$ be a finite family of semipseudo mappings such that $\cap_{i=1}^{N} F\left(T_{i}\right) \neq \emptyset$. Suppose that $s=\inf \left\{s_{i}\right\}>0$, then there exists semi-pseudo mapping $T: C \rightarrow C$ such that $F(T)=\cap_{i=1}^{m} F\left(T_{i}\right)$.

Proof Let $\left\{s_{i}\right\}$ be sequence of positive real numbers in $[0,1]$ satisfying $\sum_{i=1}^{m} s_{i}=1$ and set $T=\sum_{i=1}^{m} s_{i} T_{i}$. Since each $T_{i}$ is semi-pseudo for any $i \in\{1,2, \ldots, N\}$, and $A_{i}=J-T_{i}$ is monotone, thus we have that $T$ is well defined semi-pseudo mapping, and

$$
\begin{aligned}
\langle(J-T) x-(J-T) y, x-y\rangle & =\left\langle\left(J-\sum_{i=1}^{m} s_{i} T_{i}\right) x-\left(J-\sum_{i=1}^{m} s_{i} T_{i}\right) y, x-y\right\rangle \\
& =\left\langle\left(\sum_{i=1}^{m} s_{i}\left(J-T_{i}\right)\right) x-\left(\sum_{i=1}^{m} s_{i}\left(J-T_{i}\right)\right) y, x-y\right\rangle \\
& =\left\langle\left(\sum_{i=1}^{m} s_{i} A_{i}\right) x-\left(\sum_{i=1}^{m} s_{i} A_{i}\right) y, x-y\right\rangle \geq 0,
\end{aligned}
$$

which means that $J-T$ is monotone, therefore $T$ is semi-pseudo mapping.

Next, we claim that $F_{J}(T)=\cap_{i=1}^{N} F_{J}\left(T_{i}\right)$.

Clearly $\cap_{i=1}^{N} F\left(T_{i}\right) \subset F_{J}(T)$.

Now we prove that $F_{J}(T) \subset \cap_{i=1}^{N} F_{J}\left(T_{i}\right)$.

Let $x \in F_{J}(T)$ and $p \in \cap_{i=1}^{N} F_{J}\left(T_{i}\right)$. Then

$0=\langle T x-J x, x-p\rangle=\left\langle\sum_{i=1}^{N} s_{i} T_{i} x-J x, x-p\right\rangle=\sum_{i=1}^{N} s_{i}\left\langle T_{i} x-J x, x-p\right\rangle$, 
noticing that $A_{i}$ is monotone and $A_{i} p=0$, therefore

$$
0=\sum_{i=1}^{N} s_{i}\left\langle T_{i} x-J x, x-p\right\rangle=\sum_{i=1}^{N} s_{i}\left\langle A_{i} x-A_{i} p, x-p\right\rangle,
$$

which means that $\left\langle T_{i} x-J x, x-p\right\rangle=0$ for each $i=1,2, \cdots N$, i.e. $T_{i} x=J x$ for each $i=1,2, \cdots N$. Therefore, $x \in \cap_{i=1}^{N} F_{J}\left(T_{i}\right)$ and hence $F(T)=\cap_{i=1}^{N} F_{J}\left(T_{i}\right)$. The proof is complete.

Theorem 4.6 Let E be a nonempty closed uniformly convex and 2-uniformly smooth Banach space with dual $E^{*}$. Assume that $T_{i}: E^{*} \rightarrow E$ are a finite of $L_{i}$-Lipshitz continuous semi-pseudo mapping such that $F=\cap F_{J}\left(T_{i}\right) \neq \varnothing$ and $A:=J^{-1}-$ $\Sigma_{i=1}^{N} s_{i} T_{i}$ is maximal monotone mapping. Let $f: C \rightarrow C$ be a contraction with a coefficient $\rho \in(0,1)$. Let $\left\{x_{n}\right\}$ be a sequence generated by $x_{0} \in E$

$$
x_{n+1}=\alpha_{n} f\left(x_{n}\right)+\beta_{n} x_{n}+\gamma_{n}\left(\left(1-\omega_{n}\right) I+\omega_{n} \Sigma_{i=1}^{N} s_{i} T_{i} J\right)\left(\frac{x_{n}+x_{n+1}}{2}\right),
$$

where $J$ is the normalized duality mapping and $\left\{\omega_{n}\right\},\left\{\alpha_{n}\right\},\left\{\beta_{n}\right\},\left\{\gamma_{n}\right\}$ are sequences of nonnegative real numbers in $[0,1]$ and

(i): $\quad \alpha_{n}+\beta_{n}+\gamma_{n}=1$;

(ii): $\quad \lim _{n \rightarrow \infty} \alpha_{n}=0, \Sigma_{n=1}^{\infty} \alpha_{n}=\infty$;

(iii): $\quad \lim _{n \rightarrow \infty} \frac{\omega_{n}}{\alpha_{n}}=0$.

Suppose that $C_{\min } \cap F \neq \emptyset$, then the sequence $\left\{x_{n}\right\}$ converges strongly to an element $x^{*} \in F$.

Especially, the following corollary holds:

Theorem 4.7 Let E be a nonempty closed uniformly convex and 2-uniformly smooth Banach space with dual $E^{*}$. Assume that $T_{i}: E^{*} \rightarrow E$ is a $L_{i}$-Lipshitz continuous semi-pseudo mapping such that $F=\cap F_{J}\left(T_{i}\right) \neq \emptyset$ and $A:=J^{-1}-\Sigma_{i=1}^{N} s_{i} T_{i}$ is maximal monotone mapping. Let $f: C \rightarrow C$ be a contraction with a coefficient $\rho \in(0,1)$. Let $\left\{x_{n}\right\}$ be a sequence generated by $x_{0} \in E$

$$
x_{n+1}=\alpha_{n} u+\beta_{n} x_{n}+\gamma_{n}\left(\left(1-\omega_{n}\right) I+\omega_{n} \Sigma_{i=1}^{N} s_{i} T_{i} J\right)\left(\frac{x_{n}+x_{n+1}}{2}\right),
$$

where $J$ is the normalized duality mapping and $\left\{\omega_{n}\right\},\left\{\alpha_{n}\right\},\left\{\beta_{n}\right\},\left\{\gamma_{n}\right\}$ are sequences of nonnegative real numbers in $[0,1]$ and

(i): $\quad \alpha_{n}+\beta_{n}+\gamma_{n}=1$;

(ii): $\quad \lim _{n \rightarrow \infty} \alpha_{n}=0, \Sigma_{n=1}^{\infty} \alpha_{n}=\infty$;

(iii): $\quad \lim _{n \rightarrow \infty} \frac{\omega_{n}}{\alpha_{n}}=0$.

Suppose that $C_{\min } \cap F \neq \emptyset$, then the sequence $\left\{x_{n}\right\}$ converges strongly to an element $x^{*} \in F$. 


\section{Numerical example}

In the sequel, we give some numerical examples to illustrate the applicability, effectiveness, efficiency and stability of our semi-implicit midpoint rule(SIMR) proposed algorithm. We have written all the codes in Matlab R2016b and preformed on a LG dual core personal computer.

\subsection{Numerical behavior of SIMR}

Example 5.1 Let $E=\mathbb{R}, C=E$. Let $A, J: \mathbb{R} \rightarrow \mathbb{R}$ be the mappings defined as

$$
A x=a x^{m}, J x=x,(m=1,2),
$$

$f: C \rightarrow C$ be defined as

$$
f(x)=\left\{\begin{array}{l}
\frac{x}{2}, \text { if } A x \neq 0, \\
x, \text { if } A x=0 .
\end{array}\right.
$$

Thus, we have that $J$ is a duality mapping and for $x, y \in \mathbb{R}$,

$$
\begin{aligned}
\|A x-A y\| & =\left\|a x^{m}-a y^{m}\right\| \\
& \leq a\|x-y\|, \\
\|J x-J y\| & =\|x-y\| .
\end{aligned}
$$

Hence, $A$ is $a$-Lipschitz continuous monotone with the condition $x+y \leq 1, J$ is 1-Lipschitz continuous and $f$ is contractive with coefficient $\rho=\frac{1}{2}$ and $(A J)^{-1}(0)=0$.

Now we set $\alpha_{n}=\gamma_{n}=\frac{1}{n+1}, \beta_{n}=\frac{n-1}{n+1}, \omega_{n}=\frac{1}{n(n+1)}$, we can see all these parameters satisfy conditions:

(i) $: \alpha_{n}+\beta_{n}+\gamma_{n}=1$, also

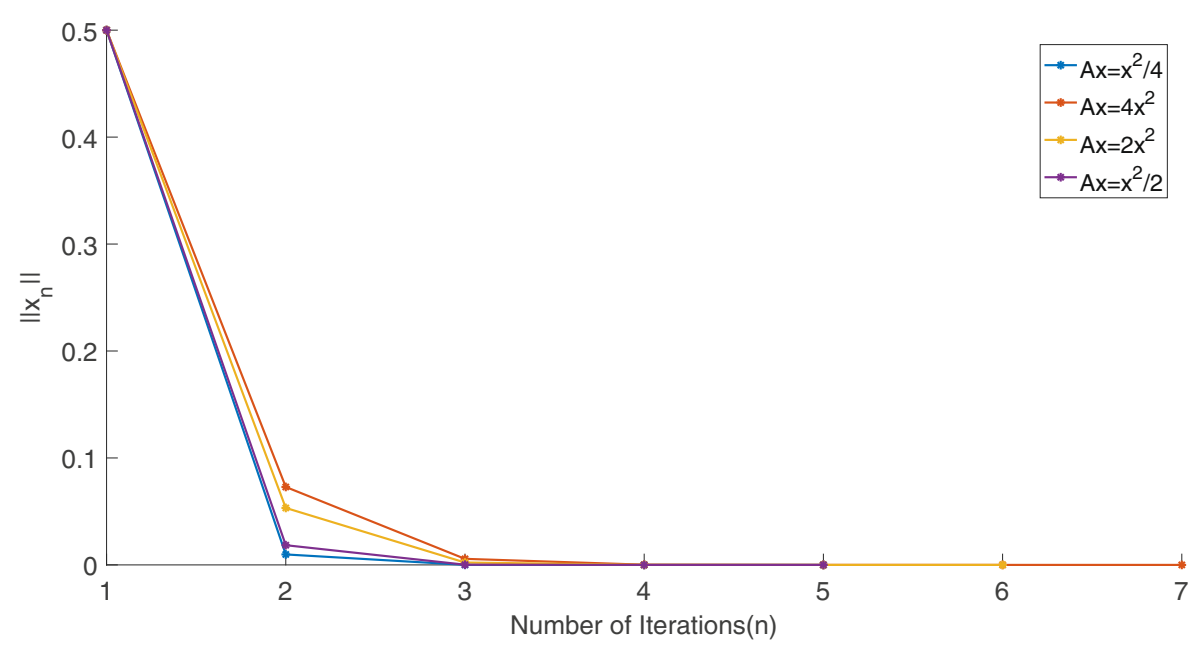

Fig. 1 The case of $x_{0}=0.5$ 


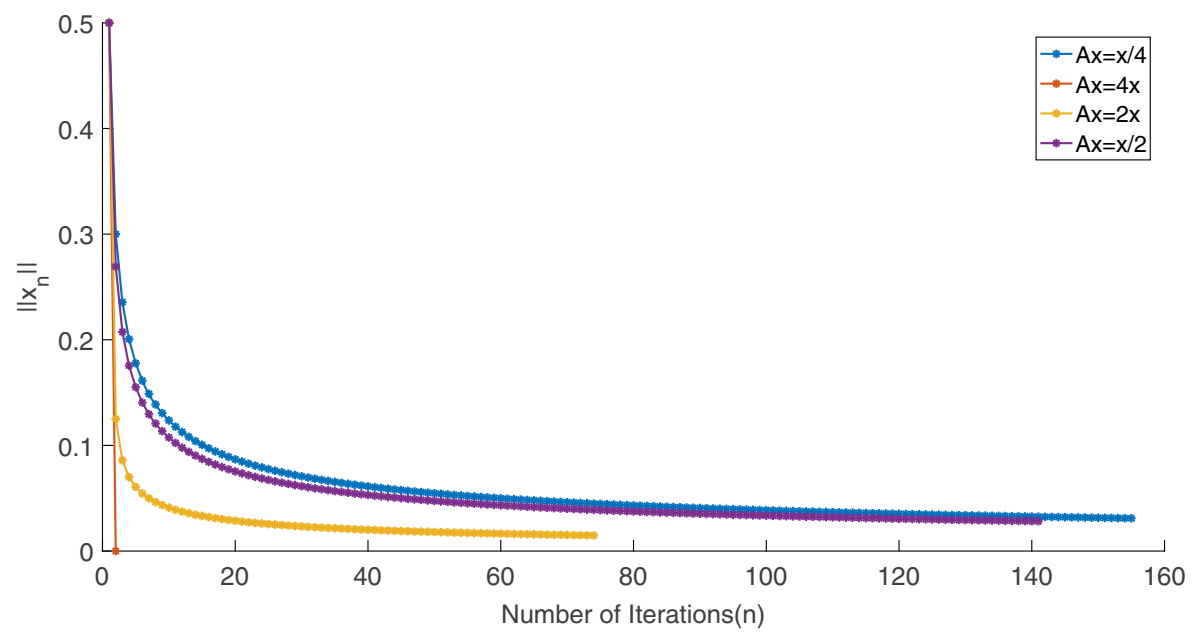

Fig. 2 The case of $x_{0}=0.5$

(ii) $: \lim _{n \rightarrow \infty} \alpha_{n}=0, \Sigma_{n=1}^{\infty} \alpha_{n}=\infty$,

(iii) $: \omega_{n}=o\left(\alpha_{n}\right)$ and $\Sigma_{n=1}^{\infty} \omega_{n}<\infty$.

In addition, it is obviously that if $x_{n} \in(A J)^{-1}(0)$, then the process stop and $x_{n}$ is the solution of problem $0 \in A u$. Otherwise, we shall compute the following semi-implicit midpoint rule

$x_{n+1}=\frac{x_{n}}{2(n+1)}+\frac{(n-1) x_{n}}{n+1}+\frac{1}{n+1}\left(\frac{x_{n}+x_{n+1}}{2}-\frac{a}{n(n+1)}\left(\frac{x_{n}+x_{n+1}}{2}\right)^{m}\right)$.

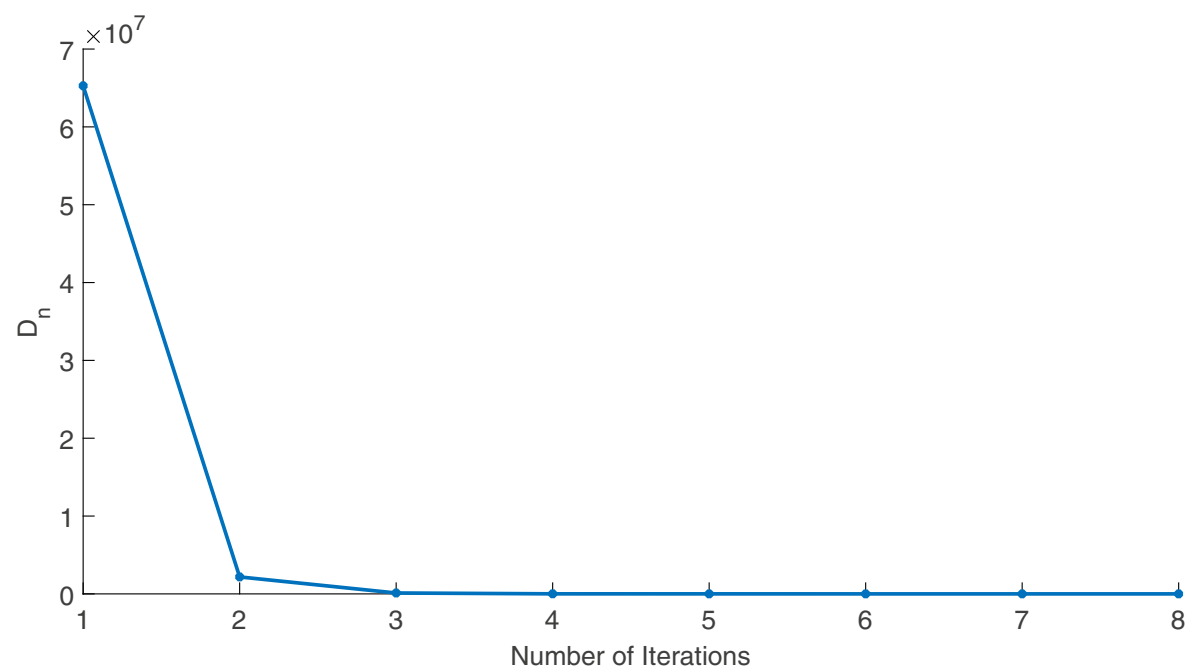

Fig. $3 D_{n}$ and Number of Iterations with $A x=\frac{x^{2}}{2}$ 


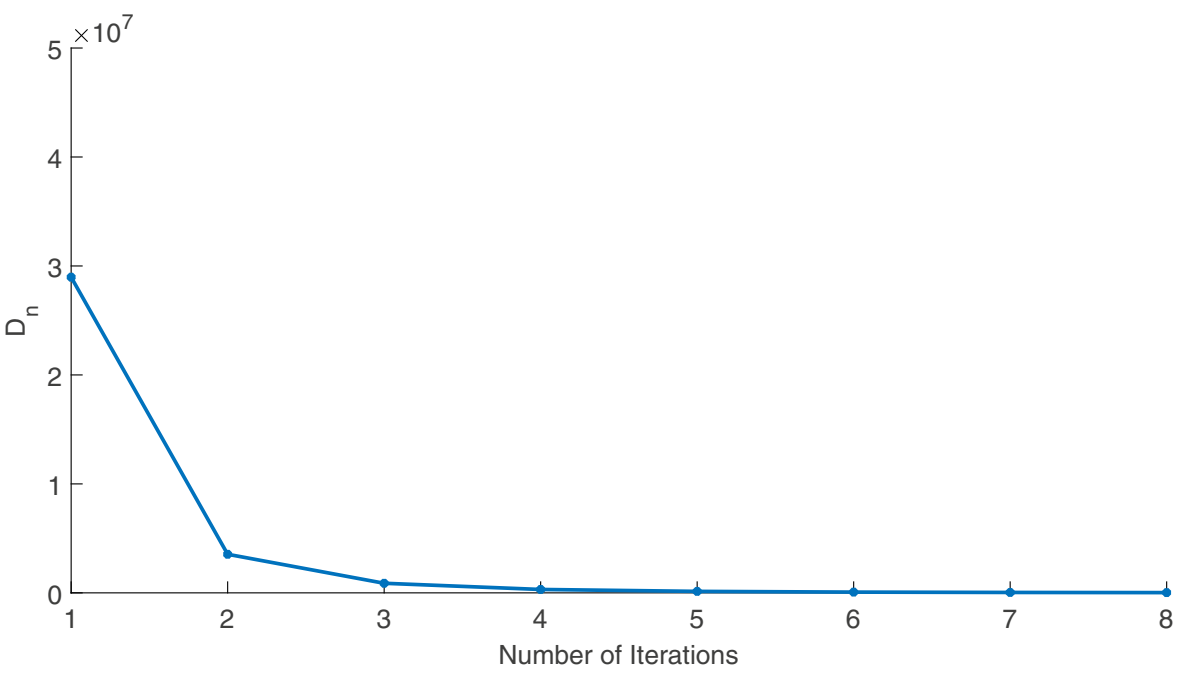

Fig. $4 D_{n}$ and Number of Iterations with $A x=\frac{x}{4}$

The convergence of the rule is illustrated in the following figures for different initial point $x_{o}$ and coefficient $a$ and $m$.

In these figures (Figs. 1, 2, 3, 4, 5, 6, 7, 8, 9, 10, 11 and 12), $x$-axes represent for the number of iterations while $y$-axes represent the value of $D_{n}$ or $x_{n}$ (where the stop criterion is $\left\|x_{n+1}-x_{n}\right\|=10^{-16}$. We can summarize the following observations from these figures:

(a) The rate of $D_{n}=10^{9}\left\|x_{n+1}-x_{n}\right\|^{2}$ generated by Algorithm 3.1 depends strictly on the convergent rate of parameter $\left\{\alpha_{n}\right\}$ and the Lipschitz continuous monotone operator.

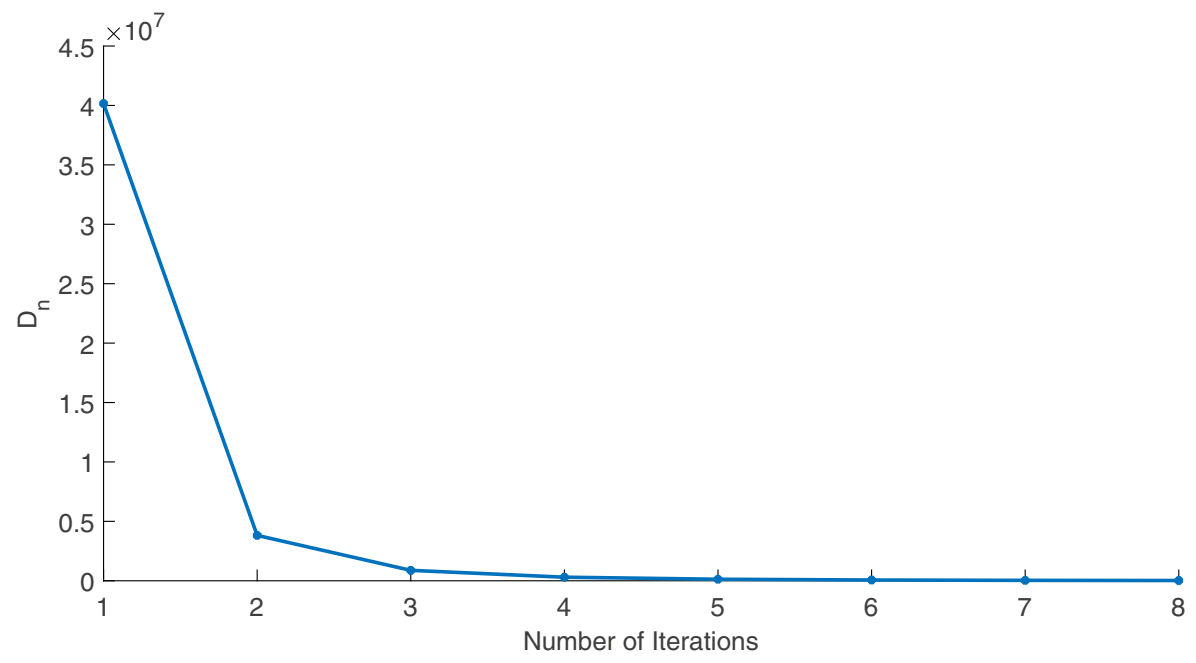

Fig. $5 D_{n}$ and Number of Iterations with $A x=\frac{x^{2}}{4}$ 


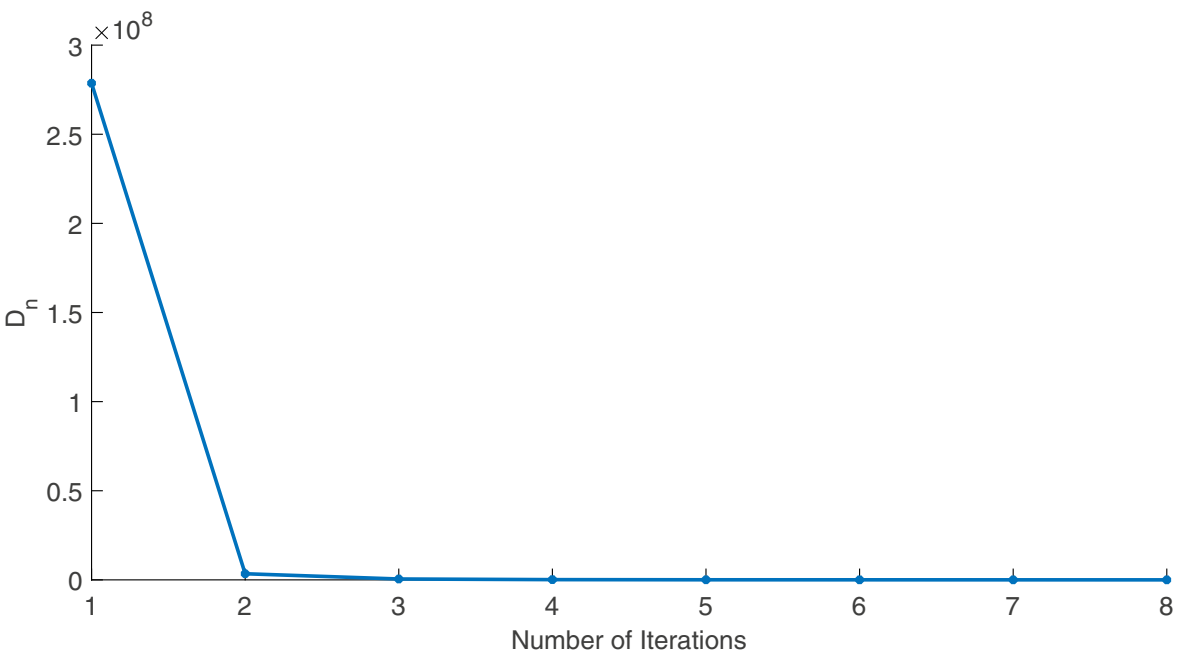

Fig. $6 D_{n}$ and Number of Iterations with $A x=4 x^{2}$

(b) In general, Algorithm 3.1 works well for the parameter $\left\{\alpha_{n}\right\}$ being fast convergent to 0 as $n \rightarrow \infty$. The best error of $D_{n}$ can be obtained approximately equal to $10^{-16}$. When $D_{n}$ obtains to this error, it will become unstable.

Example 5.2 For the uniformly convex and uniformly smooth Banach spaces $\ell^{p}$, we can recall from Alber [33] the example of the normalized duality mapping $J$ as

$$
J x=\|x\|_{\ell p}^{2-p} y,
$$

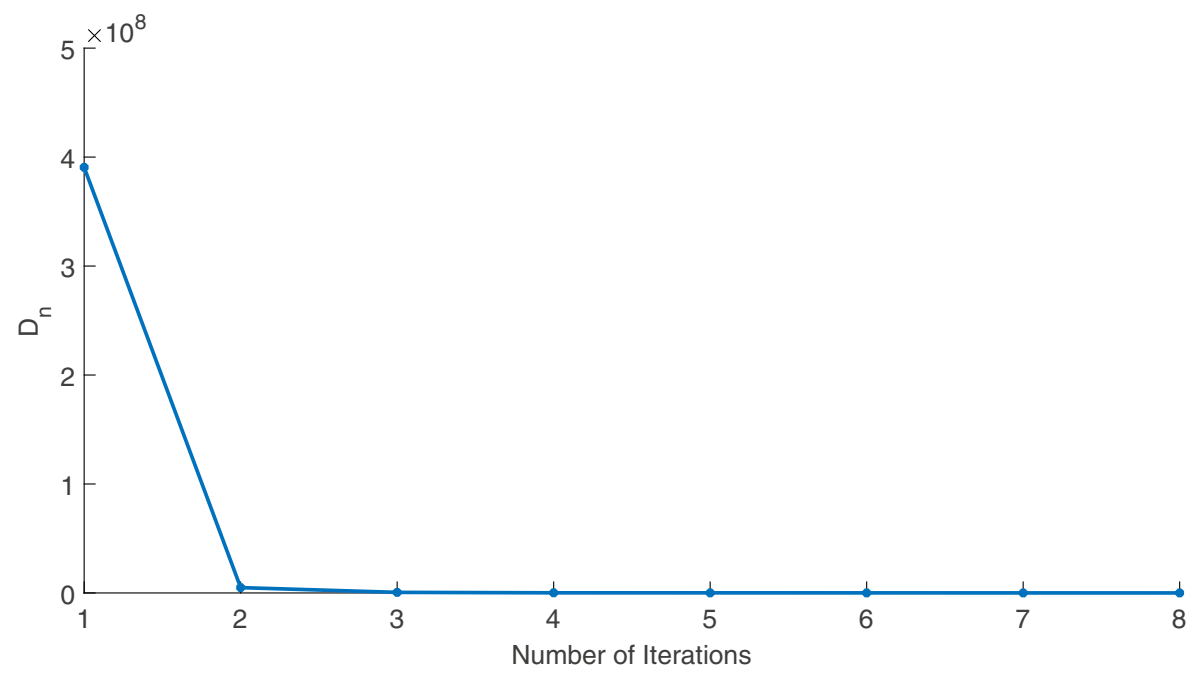

Fig. $7 D_{n}$ and Number of Iterations with $A x=4 x$ 


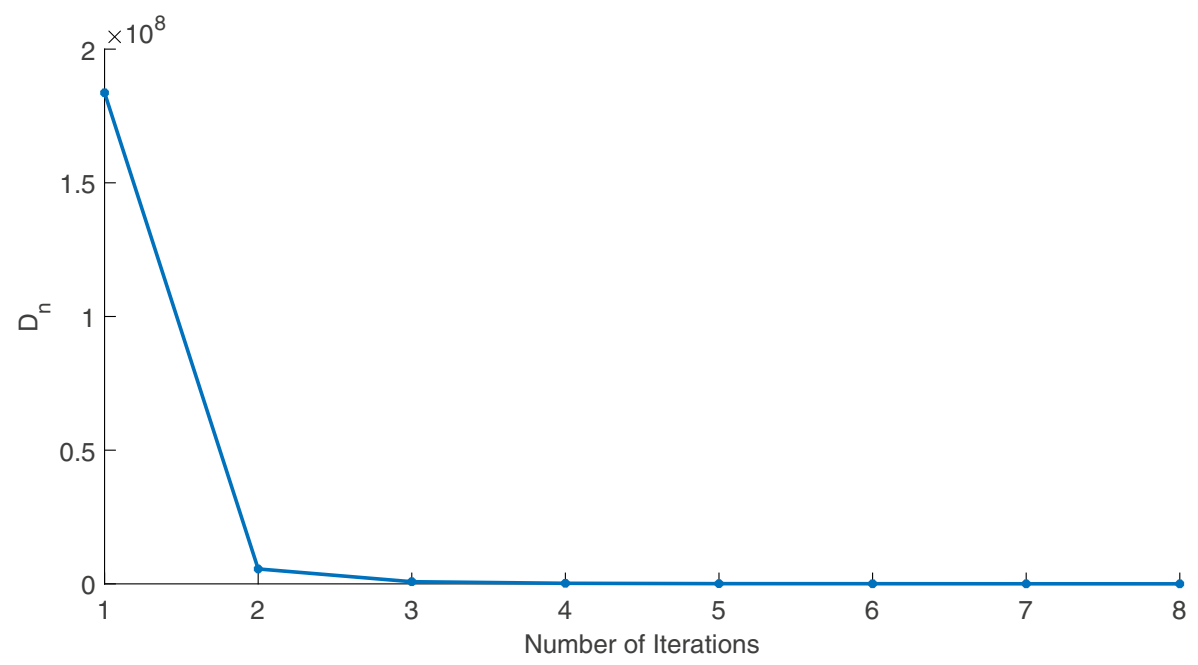

Fig. $8 D_{n}$ and Number of Iterations with $A x=2 x$

where $x=\left\{x_{1}, x_{2}, x_{3}, \ldots\right\}, y=\left\{x_{1}\left|x_{1}\right|^{p-2}, x_{2}\left|x_{2}\right|^{p-2}, x_{3}\left|x_{3}\right|^{p-2}, \ldots\right\} \in \ell^{q}$ with $\frac{1}{p}+\frac{1}{q}=1$ and norm $\|\cdot\|_{\ell}=\left(\Sigma_{n=1}^{\infty} x_{n}^{p}\right)^{\frac{1}{p}}$. As the reviewers mention, the duality mapping $J$ is not easy to compute, hence for simplicity, we take $p=2$ and define $A x=\frac{x}{2}$ and $f: C \rightarrow C$ as

$$
f(x)=\left\{\begin{array}{l}
\frac{x}{2}, \text { if } A x \neq 0 \\
x, \text { if } A x=0
\end{array}\right.
$$

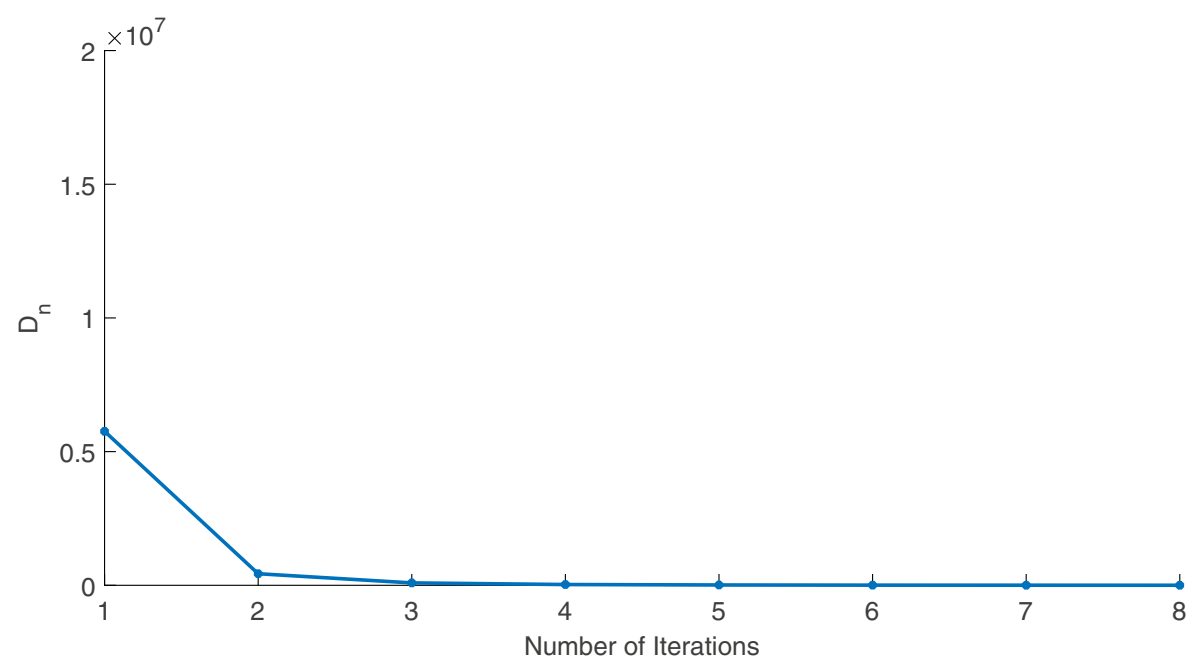

Fig. $9 D_{n}$ and Number of Iterations with $A x=\frac{x}{2}$ 


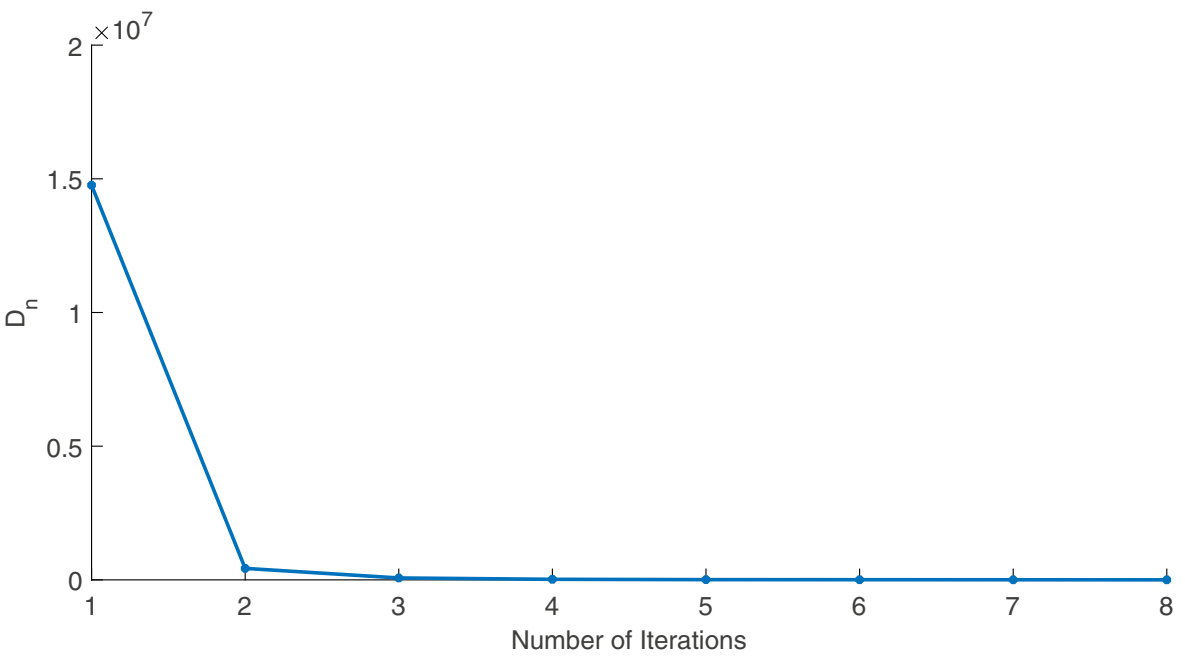

Fig. $10 D_{n}$ and Number of Iterations with $A x=2 x^{2}$

The parameters $\alpha_{n}=\frac{1}{n+1}, \beta_{n}=0$ and $\omega_{n}=\frac{1}{n(n+1)}$, if $x_{n} \in(A J)^{-1}(0)$, then the process stop and $x_{n}$ is the solution of problem $0 \in A u$. Otherwise, we shall compute the following semi-implicit midpoint rule

$$
x_{n+1}=\frac{x_{n}}{2(n+1)}+\frac{n}{n+1}\left(\frac{x_{n}+x_{n+1}}{2}-\frac{1}{2 n(n+1)}\left(\frac{x_{n}+x_{n+1}}{2}\right)\right) .
$$

We test the initial point $x_{0}=\{1,0,0, \ldots\}$ for our Algorithm 3.1 and the test results are reported in Table 1.

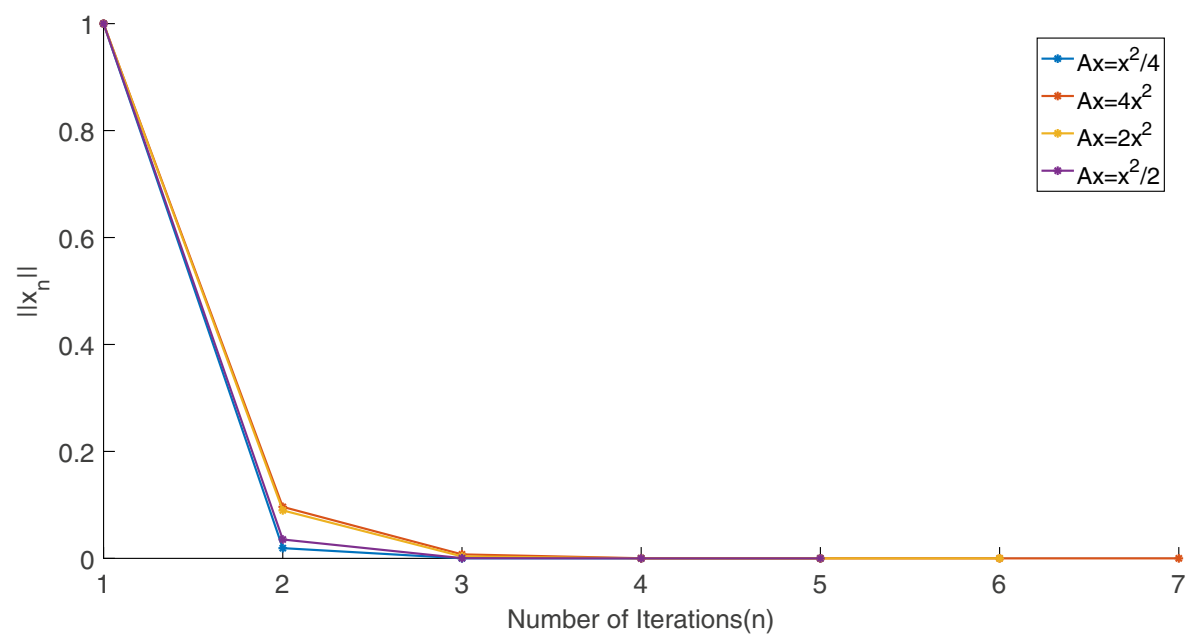

Fig. 11 the case of $x_{0}=1$ 


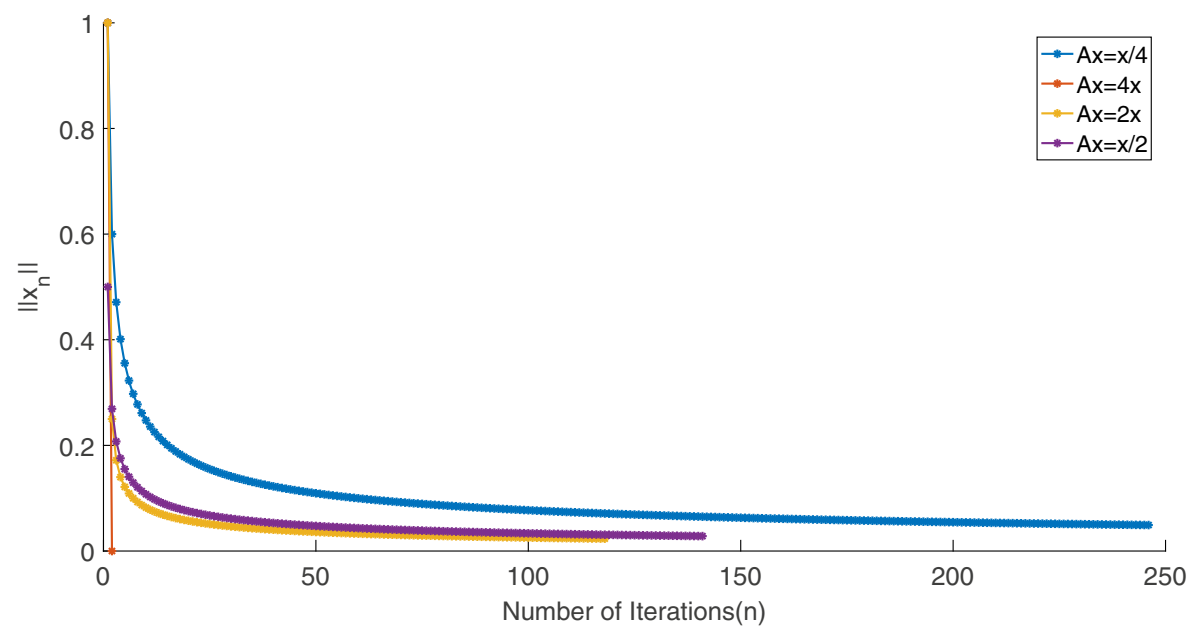

Fig. 12 the case of $x_{0}=1$

Table 1 Comparison between Algorithm 3.1 and other algorithms $x_{0}=(1,0,0,0,0,0, \ldots)$

\begin{tabular}{lllll}
\hline $\mathrm{n}$ & $x_{n+1}$ & $x_{n}$ & $\left\|x_{n+1}-x_{n}\right\|$ & $\left\|x_{n}\right\|$ \\
\hline 0 & $(0.5385,0,0,0,0,0, \ldots)$ & $(1,0,0,0,0,0, \ldots)$ & 0.4615 & 1 \\
1 & $(0.3662,0,0,0,0,0, \ldots)$ & $(0.5385,0,0,0,0,0, \ldots)$ & 0.1723 & 0.5385 \\
10 & $(0.0933,0,0,0,0,0, \ldots)$ & $(0.1017,0,0,0,0,0, \ldots)$ & 0.0084 & 0.1017 \\
30 & $(0.0350,0,0,0,0,0, \ldots)$ & $(0.0362,0,0,0,0,0, \ldots)$ & 0.0012 & 0.0362 \\
100 & $(0.0110,0,0,0,0,0, \ldots)$ & $(0.0111,0,0,0,0,0, \ldots)$ & 0.0012 & 0.0362 \\
330 & $(0.0034,0,0,0,0,0, \ldots)$ & $(0.0034,0,0,0,0,0, \ldots)$ & 0.0000 & 0.0034 \\
\hline
\end{tabular}

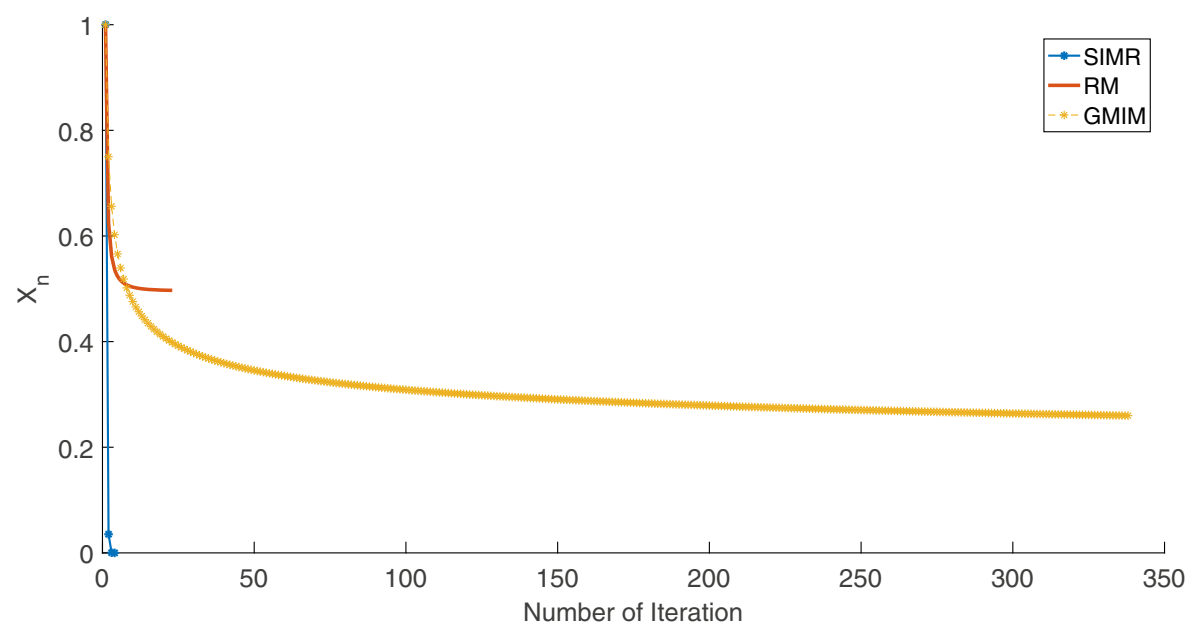

Fig. 13 Comparison of SIMR,RM,GMIM for $A x=\frac{x^{2}}{2}$ 


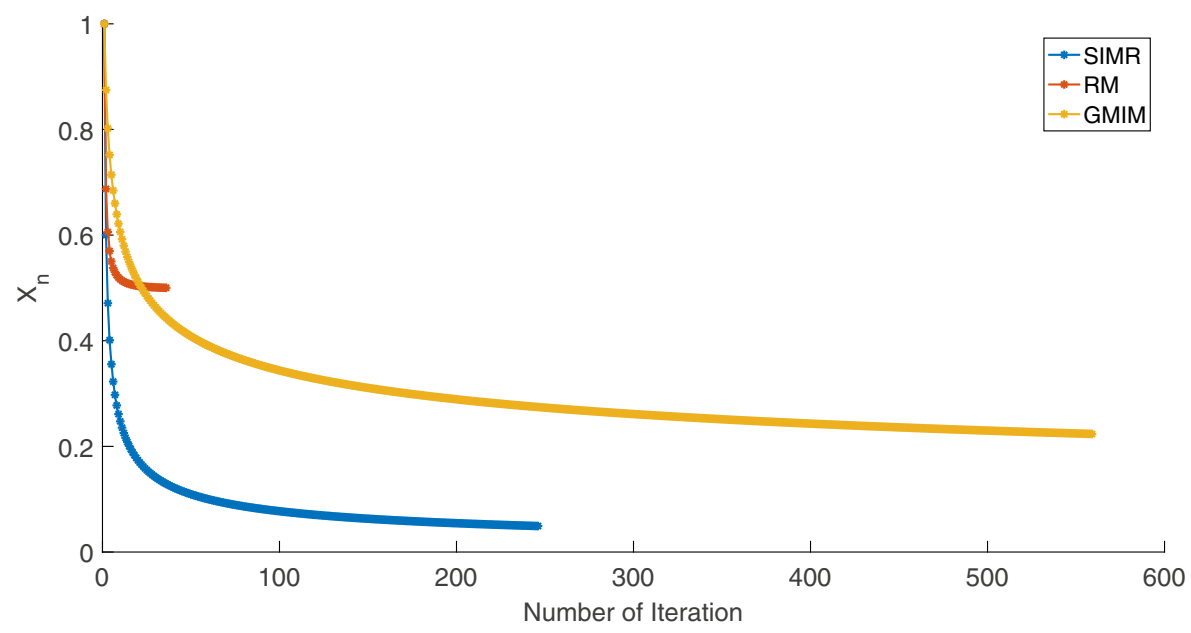

Fig. 14 Comparison of SIMR,RM,GMIM for $A x=\frac{x}{4}$

From this example, we can obtain the sequence converges to one element of $(A J)^{-1}(0)$.

\subsection{Comparison SIMR with other algorithms}

In this part, we present several experiments in comparison with other algorithms. Two methods used to compare are generalized mann iteration method (GMIM)( [29],

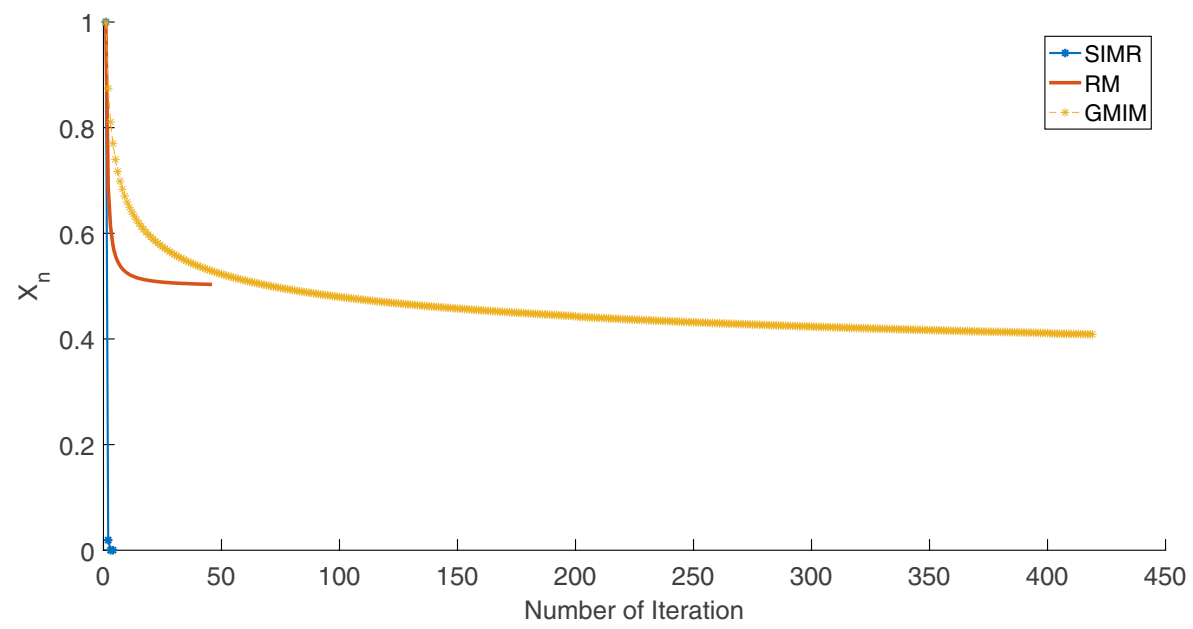

Fig. 15 Comparison of SIMR,RM,GMIM for $A x=x^{2} / 4$ 
Table 2 Comparison between Algorithm 3.1 and other algorithms $x_{0}=1$

\begin{tabular}{lllllll}
\hline TOL & SIMR(Iter) & CPU(s) & RM(Iter)(u=1) & CPU(s) & GMIM(Iter) & CPU(s) \\
\hline $10^{-8}$ & 56 & 0.079 & 83 & 0.0608 & 419 & 0.073 \\
$10^{-10}$ & 175 & 0.084 & 319 & 0.0909 & 2919 & 0.252 \\
$10^{-12}$ & 307 & 0.102 & 1156 & 0.179 & 21331 & 1.96 \\
TOL & SIMR(Iter) & CPU(s) & RM(Iter)(u=0.5) & CPU(s) & GMIM(Iter) & CPU(s) \\
$10^{-8}$ & 56 & 0.079 & 46 & 0.063 & 419 & 0.073 \\
$10^{-10}$ & 176 & 0.084 & 123 & 0.065 & 2919 & 0.252 \\
$10^{-12}$ & 307 & 0.102 & 307 & 0.095 & 21331 & 1.96 \\
\hline
\end{tabular}

Algorithm 1), regularization method (RM)( [22], Algorithm 2). RM require to previously know a constant $u$. For experiments, we choose the same sequence $\alpha_{n}=\frac{1}{n+1}$ and $\omega_{n}=\frac{1}{n(n+1)}$ in GMIM and RM. The condition $\left\|x_{n+1}-x_{n}\right\|^{2} \leq T O L$ is chosen to be as the stopping criterion. The following figures (Figs. 13, 14, and 15) are comparisons of SIMR, RM, GMIM with different monotone operators.

The numerical results are showed in Tables 2 and 3 with different initial value and $u$.

From these tables, we can see that our Algorithm 3.1 (SIMR) is the best. The GMIM is the most time-consuming and the reasonable explanation is the fact that at each step the GMIM has no the contractive parameters (coefficients) for obtaining the next step which can take lower convergence rate, while the convergent rate of RM depends strictly on the previous constant $u$ and the initial value $x_{0}$. In comparing with other two methods, our Algorithm 3.1 seems to have a competitive advantage. However, the main advantage of our Algorithm 3.1 is that the semi-implicit midpoint rule works more stable than other methods and it is done in Banach spaces much more general than Hilbert spaces.

Table 3 Comparison between Algorithm 3.1 and other algorithms $x_{0}=0.5$

\begin{tabular}{lllllll}
\hline TOL & SIMR(Iter) & CPU(s) & RM(Iter)(u=1) & CPU(s) & GMIM(Iter) & CPU(s) \\
\hline $10^{-8}$ & 40 & 0.074 & 103 & 0.065 & 233 & 0.063 \\
$10^{-10}$ & 127 & 0.075 & 371 & 0.100 & 1749 & 0.163 \\
$10^{-12}$ & 269 & 0.085 & 1300 & 0.203 & 13569 & 1.141 \\
TOL & SIMR(Iter) & CPU(s) & RM(Iter)(u=0.5) & CPU(s) & GMIM(Iter) & CPU(s) \\
$10^{-8}$ & 40 & 0.074 & 36 & 0.059 & 233 & 0.063 \\
$10^{-10}$ & 127 & 0.075 & 146 & 0.064 & 1749 & 0.163 \\
$10^{-12}$ & 269 & 0.085 & 544 & 0.112 & 13569 & 1.141 \\
\hline
\end{tabular}




\section{Conclusion}

Let $E$ be a nonempty closed uniformly convex and 2-uniformly smooth Banach space with dual $E^{*}$. Approximation of zero points of accretive mappings has been explored with the past several decades. The key method of this study is to approximate the fixed point of pseudo-contractive mappings because the operator $A:=I-T$ is accretive if and only $T$ is pseudo-contractive.

However, the technique of converting the inclusion $0 \in A u$ into a fixed point problem for $(I-A): E \rightarrow E$ is not applicable since, in this case when $A$ is monotone, $A$ maps $E$ into $E^{*}$, and the identity map does not make sense.

This motivated the study of zeros of monotone mappings via semi-fixed point. The main result of this paper is to study the semi-implicit midpoint rule with the viscosity methods in Banach spaces which is a easily applicable iterative algorithm that converges strongly to zero point of monotone mapping. Although the proof of the main result is very technical and nontrivial, with the simple restriction of parameters, the recursion formula of the theorem (Theorem 3.1) does not involve the resolvent operator which is computational complexity.

Furthermore, the theorems in this paper complement the implicit midpoint rule and proximal point algorithm by proposing strong convergence to zero of monotone mapping and extend and unify some results (see, e.g., Zegeye [22], Chidume [24], $\mathrm{Xu}$ [21]). In addition, it is applied to convex minimization problems, solution of Hammerstein integral equations and semi-fixed point of a cluster of semi-pseudo mappings. This may be the topic of some of our forthcoming papers.

Acknowledgements The authors express their deep gratitude to the referees and the editor for their valuable comments and suggestions.

Author Contributions All authors contributed equally to this work. All authors read and approved final manuscript.

Funding information This article was funded by the National Science Foundation of China (11471059)and Science and Technology Research Project of Chongqing Municipal Education Commission (KJ1706154)and the Research Project of Chongqing Technology and Business University (KFJJ2017069).

\section{Compliance with Ethical Standards}

Competing Interests The authors declare that they have no competing interests.

Open Access This article is distributed under the terms of the Creative Commons Attribution 4.0 International License (http://creativecommons.org/licenses/by/4.0/), which permits unrestricted use, distribution, and reproduction in any medium, provided you give appropriate credit to the original author(s) and the source, provide a link to the Creative Commons license, and indicate if changes were made.

\section{References}

1. Kato, T.: Nonlinear semigroups and evolution equations. J. Math. Soc. Jpn. 19, 508-520 (1967) 
2. Browder, F.E.: Nonlinear mappings of nonexpansive and accretive-type in Banach spaces. Bull. Am. Math. Soc. 73, 875-882 (1967)

3. Minty, G.J.: Monotone (nonlinear) operators in Hilbert space. Duke. Math. J. 29(4), 341-346 (1962)

4. Turkyilmazoglua, M.: Approximate analytical solution of the nonlinear system of differential equations having asymptotically stable equilibrium. Filomat 31(9), 2633-2641 (2017)

5. Duffull, S.B., Hegarty, G.: An Inductive Approximation to the Solution of Systems of Nonlinear Ordinary Differential Equations in Pharmacokinetics-Pharmacodynamics. Journal of Computer Science and Networking pp. 1-14 (2014)

6. Khorasani, S., Adibian, A.: Alytical solution of linear ordinary differential equations by differential transfer. Electronic Journal of Differential Equations 79, 1-18 (2003)

7. Chidume, C.E., Osilike, M.O.: Iterative solution of nonlinear integral equations of Hammerstein-type. J. Niger. Math. Soc. Appl. Anal. pp. 353-367 (2003)

8. Auzinger, W., Frank, R.: Asyptotic error expansions for stiff equations: an analysis for the implicit midpoint and trapezoidal rules in the strongly stiff case, vol. 56 (1989)

9. Bader, G., Deuflhard, P.: A semi-implicitmid-point rule for stiff systems of ordinary differential equations. Number. Math. 41, 373-398 (1983)

10. Bayreuth, A.: The implicit midpoint rule applied to discontinuous differential equations. Computing 49, 45-62 (1992)

11. Xu, H.K., Alghamdi, M.A., Shahzad, N.: The viscosity technique for the implicit midpoint rule of nonexpansive mappings in Hilbert spaces. Fixed Point Theory Appl. 2015, 41 (2015)

12. Berinde, V.: Iterative approximation of fixed points. Lecture Notes in Mathematics. Springer, London (2007)

13. Browder, F.E.: Nonlinear mappings of nonexpansive and accretive-type in Banach spaces. Bull. Am. Math. Soc. 73, 875-882 (1967)

14. Chidume, C.E.: Geometric Properties of Banach Spaces and Nonlinear Iterations Lectures Notes in Mathematics, vol. 1965. Springer, London (2009)

15. Chidume, C.E.: Iterative approximation of fixed points of Lipshitzian strictly pesudo-contractive mappings. Proc. Amer. Math. Soc. 99(2), 283-288 (1987)

16. Chidume, C.E., Osilike, M.O.: Iterative solutions of nonlinear accretive operator equations in arbitrary Banach spaces. Nonlinear Anal. Theory Methods Appl. 36, 863-872 (1999)

17. Agarwal, R.P., Meehan, M., O'Regan, D.: Fixed point theory and applications. Cambridge Tracts in Mathematics, vol. 141. Cambridge University Press, Cambridge (2001)

18. Chidume, C.E.: Anapproximation method for monotone Lipschitzian operators in Hilbert-spaces. J. Aust. Math. Soc. Ser. A. 41, 59-63 (1986)

19. Reich, S.: A weak convergence theorem for alternating methods with Bergman distance. In: Kartsatos, A.G. (ed.) Theory and Applications of Nonlinear Operators of Accrective and Monotone Type. Lecture Notes in Pure and Appl. Math, vol. 178, pp. 313-318. Dekker, New York (1996)

20. Chidume, C.E., Djitte, N.: Strong convergence theorems for zeros of bounded maximal monotone nonlinear operators. Abstr. Appl. Anal. 2012 Article ID 681348 (2012)

21. Xu, H.K.: An iterative approach to quadratic optimization. Journal of Optim Theory and Application 116, 659-678 (2003)

22. Zegeye, H.: Strong convergence theorems for maximal monotone mappings in Banach spaces. Journal of Mathematical Analysis and Applications 343(2), 663-671 (2008)

23. Ibaraki, T., Takashiwa, W.: A new projection and convergence theorems for the projections in Banach spaces. Journal of Approximation Theory 1-14, 149 (2007)

24. Chidume, C.E., Idu, K.O.: Approximation of zeros of bounded maximal monotone mappings, solutions of Hammerstein integral equations and convex minimization problems. Fixed Point Theory and Applications:97. https://doi.org/10.1186/s13663-016-0582-8 (2016)

25. Alber, Y.A.: Metric and generalized projection operators in Banach spaces: properties and applications. In: Theory and Applications of Nonlinear Operators of Accretive and Monotone Type, pp. 15-50. Marcel Dekker, New York (1996)

26. Takahashi, W.: Nolinear Functional Analysis. Yokohama Publishers, Yokohama (2000)

27. Tan, K.K., Xu, H.K.: Approximating fixed points of nonexpansive mappings by the Ishikawa iteration process. J. Math. Anal. Appl. 178(2), 301-308 (1993)

28. Chidume, C.E., Zegeye, H.: Approximation of solutions of nonlinear equations of monotone and Hammerstein-type. Appl. Anal. 82(8), 747-758 (2003) 
29. Chidume, C.E., Romanus, O.M., Nnyaba, U.V.: A new iterative algorithm for zeros of generalized Phistrongly monotone and bounded maps with application. British Journal of Mathematics and Computer science 18(1), 1-14 (2016)

30. Xu, H.K., Alghamdi, M.A., Shahzad, N.: The implicit midpoint rule for nonexpansive mappings in banach spaces. Fixed Point Theory 17(2), 509-517 (2016)

31. Song, Y.L., Pei, Y.G.: A new modified semi-implicit midpoint rule for nonexpansive mappings and 2generalized hybrid mappings. Journal of Nonlinear Science and Applications 9(12), 6348-6363 (2016)

32. Cai, G., Shehu, Y., Iyiola, O.S.: Strong convergence results for variational inequalities and fixed point problems using modified viscosity implicit rules. Numerical Algorithms 77(2), 535C558 (2018)

33. Alber, Y.I.: Metric and generalized projection operators in Banach spaces: properties and applications, Theory and Applications of Nonlinear Operators of Accretive and Monotone Type of Lecture Notes in Pure and Applied Mathematics. In: Kartsatos, A.G. (ed.), vol. 178, p. 15C50. Marcel Dekker, New York (1996)

34. Chidume, C.E., Idu, K.O.: Approximation of zeros of bounded maximal monotone mappings, solutions of Hammerstein integral equations and convex minimization problems. Fixed Point Theory and Applications. 2016, 97 (2016) 